Document downloaded from:

http://hdl.handle.net/10251/68034

This paper must be cited as:

Lorente, D.; Aleixos Borrás, MN.; Gómez Sanchís, J.; Cubero, S.; García Navarrete, OL.; Blasco Ivars, J. (2011). Recent advances and applications of hyperspectral imaging for fruit and vegetable quality assessment. Food and Bioprocess Technology. 5(4):1121-1142. doi:10.1007/s11947-011-0725-1.

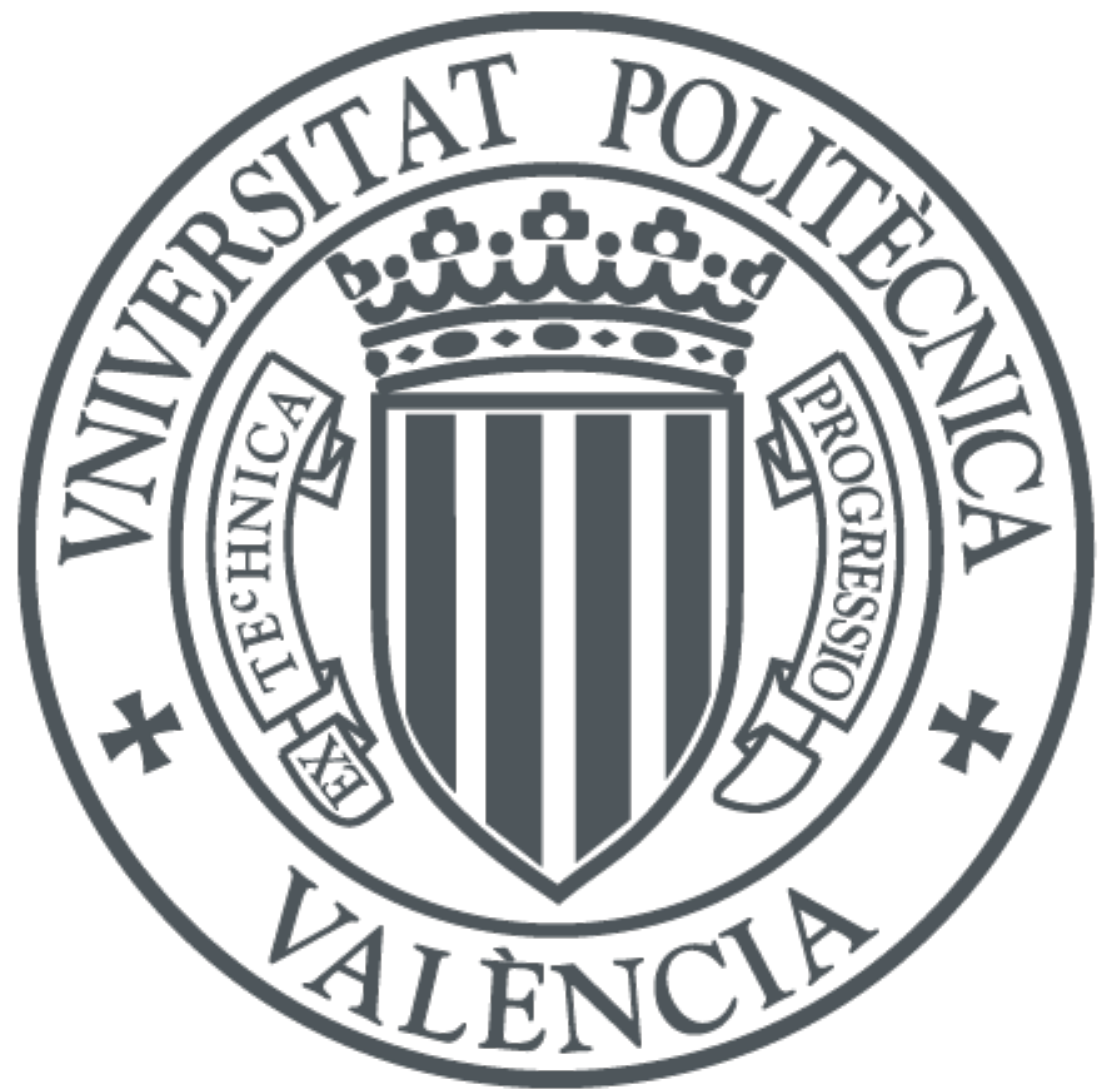

The final publication is available at

http://dx.doi.org/10.1007/s11947-011-0725-1

Copyright Springer Verlag

Additional Information 


\title{
Recent advances in hyperspectral imaging for fruit and vegetable quality assessment
}

\author{
D. Lorente ${ }^{1}$, N. Aleixos ${ }^{2}$, J. Gómez-Sanchis ${ }^{3}$, S. Cubero ${ }^{1}$, O.L. García-Navarrete ${ }^{1,4}$, \\ J. Blasco 1
}

${ }^{1}$ Centro de Agroingeniería. Instituto Valenciano de Investigaciones Agrarias. Cra. Moncada-Náquera, Km. 5, 46113 Moncada, Spain. blasco_josiva@gva.es

${ }^{2}$ Instituto Interuniversitario de Investigación en Bioingeniería y Tecnología Orientada al Ser Humano. Universitat Politècnica de València. Camino de Vera s/n, 46022 Valencia, Spain. naleixos@dig.upv.es

${ }^{3}$ Intelligent Data Analysis Laboratory, IDAL. Electronic Engineering Department. Universitat de València. Dr. Moliner 50, 46100 Burjassot (Valencia), Spain.

${ }^{4}$ Departamento de Ingeniería Civil y Agrícola. Universidad Nacional de Colombia - Sede Bogotá. Carrera 30 No. 45-03, Edificio 214, Oficina 206. Bogotá, Colombia

\begin{abstract}
Hyperspectral imaging systems are starting to be used as a scientific tool for food quality assessment. A typical hyperspectral image is composed of a set of a relatively wide range of monochromatic images corresponding to continuous wavelengths that normally contain redundant information or may exhibit a high degree of correlation. In addition, computation of the classifiers used to deal with the data obtained from the images can become excessively complex and time consuming for such high dimensional data sets and this makes it difficult to incorporate such systems into an industry that demands standard protocols or high-speed processes. Therefore, recent works have focused on the development of new systems based on this technology that are capable of analysing quality features that cannot be inspected using visible imaging. Many of those studies have also centred on finding new statistical techniques to reduce the hyperspectral images to multispectral ones, which are easier to implement in automatic, nondestructive systems. This article reviews recent works that use hyperspectral imaging for the inspection of fruit and vegetables. It explains the different technologies available to acquire the images and their use for the non-destructive inspection of internal and external features of these products. Particular attention is paid to the works aimed at reducing the dimensionality of the images, with details of the statistical techniques most commonly used for this task.
\end{abstract}

Keywords Computer vision, fruits, vegetables, quality, non-destructive inspection, image analysis, hyperspectral imaging, multispectral imaging

$\begin{array}{ll}\text { Nomenclature } & \\ \text { 2D } & \text { 2 dimensional } \\ \text { ANN } & \text { Artificial neural networks } \\ \text { ANOVA } & \text { Analysis of variance } \\ \text { AOTF } & \text { Acousto-optic tunable filters } \\ \text { BMP } & \text { Bitmap image format } \\ \text { CCD } & \text { Charge-coupled device } \\ \text { FLD } & \text { Fisher's linear discriminant } \\ \text { FWHM } & \text { Full width at half maximum } \\ \text { GALDA } & \text { Genetic algorithm based on LDA } \\ \text { LCTF } & \text { Liquid crystal tunable filters } \\ \text { LD } & \text { Lorentzian distribution } \\ \text { LDA } & \text { Linear discriminant analysis } \\ \text { MC } & \text { Moisture content } \\ \text { MD } & \text { Mahalanobis distance } \\ \text { NIR } & \text { Near-infrared } \\ \text { PCA } & \text { Principal component analysis } \\ \text { PLS } & \text { Partial least square } \\ \text { PLSDA } & \text { PLS discriminant analysis } \\ \text { PLSR } & \text { PLS regression } \\ \text { RF } & \text { Radio frequency }\end{array}$




$\begin{array}{ll}\text { RGB } & \text { Red, Green, Blue colour space } \\ \text { RGBI } & \text { Red, Green, Blue, Infrared } \\ \text { SAM } & \text { Spectral angle mapper } \\ \text { SID } & \text { Spectral information divergence } \\ \text { SSC } & \text { Soluble solids content } \\ \text { TA } & \text { Titratable acid } \\ \text { UV } & \text { Ultraviolet }\end{array}$

\section{Introduction}

The application of machine vision to food analyses has increased considerably in recent years, and has been used with meat (Du \& Sun, 2009), fish (Quevedo et al., 2010; Quevedo \& Aguilera, 2010), grains (Manickavasagan et al., 2010), bread (Farrera-Rebollo et al., 2011), fruits and vegetables (Cubero et al., 2011), among others. The breadth of applications depends, among many other things, on the fact that machine vision systems provide substantial information about the nature and attributes of the objects present in a scene. Another important feature of such systems is that they open up the possibility of studying these objects in regions of the electromagnetic spectrum where the human eye is unable to operate, such as in the ultraviolet (UV), near-infrared (NIR) or infrared (IR) regions.

The high risk of human error in classification processes has been underlined and is one of the most important drawbacks that machine vision can help prevent. In a study carried out with different varieties of apples, where various shape, size and colour parameters were compared, one of the conclusions reached was the limited human capacity to reproduce the estimation of quality, which is defined as inconsistency (Paulus et al., 1997). Moreover, as the number of parameters considered in the decisionmaking process increases, so does the rate of error in classification. Furthermore, it should also be mentioned that automatic inspection allows precise statistics to be generated on aspects related to the quality of the inspected product, which leads to greater control over it and facilitates its traceability.

In this respect, the quality of a particular fresh or processed fruit or vegetable is defined by a series of external characteristics that make it more or less attractive to the consumer. Such attributes include its ripeness, size, weight, shape, colour, the presence of blemishes and disease, the presence or absence of fruit stems, the presence of seeds, and so on, as well as a series of internal properties like sweetness, acidity, texture, hardness, etc. that can influence the consumer's decision as to whether to repeat the consumption of a particular fruit or not. In sum, they cover all of the factors that exert an influence on the product's appearance, on its nutritional and organoleptic qualities or on its suitability for preservation. Most of these factors have traditionally been assessed by visual inspection or destructive sampling performed by trained operators, but currently many of them, particularly the external ones, can be estimated with commercial vision systems (Cubero et al., 2011). These vision systems for fruit sorting are normally based on colour video cameras that imitate the vision of the human eye by capturing images using three filters centred on red, green and blue (RGB) wavelengths. Therefore, they are limited to observing scenes and are usually incapable of obtaining much information about the external or internal composition of the products.

One way to enhance the capability of traditional colour systems that seek to imitate the human eye is the use of multispectral systems. A hyperspectral image is composed of a relatively wide range of continuous wavelengths, whereas a multispectral image consists of a few wavelengths that do not necessarily have to be continuous. The main advantages of multispectral imaging systems are the relatively low cost of the system in comparison with hyperspectral systems and the fact that they can be more specific for real applications. In fact, hyperspectral systems are sometimes used just to select the particular set of wavelengths that will finally be used. An RGB camera could be considered a particular case of a multispectral system although it is more common to include wavelengths in frequencies outside the visible, like NIR. For instance, Aleixos et al. (2002) developed a multispectral camera for the inspection of citrus fruits which was able to acquire visible and near-infrared images (RGBI) from the same scene. The same authors also developed specific algorithms for inspecting the size, colour and presence of defects in citrus at a rate of between 5 and 10 fruits/s. The camera had two CCDs, one of which was a colour CCD that provided RGB information and the other was monochromatic but coupled to an IR filter, centred on $750 \mathrm{~nm}$, which provided IR information. For defect detection, a Bayesian discriminant model was used to segment the images at the pixel level, the independent variables being the grey levels of the RGBI bands and the classes background, defect and sound skin. The experiments were carried out with oranges, mandarins and lemons. Comparing results with those obtained using human classification showed $94 \%$ coincidence in the worst case (when the fruit was changing colour from green to orange). 
The system was also capable of correctly classifying lemons and mandarins, and detected the external defects in $93 \%$ and $94 \%$ of cases, respectively. One of the conclusions was that the B improved the detection of defects compared to using only RGI, but its contribution was of little importance. Taghizadeh et al. (2011) compared a conventional RGB imaging system based on a standard still camera with a hyperspectral imaging system $(400-1000 \mathrm{~nm})$ to evaluate the quality of mushrooms by estimating the hunter L-value, which is the most commonly applied feature for mushroom quality grading. Different model performance indicators showed the reasonably high potential of hyperspectral imaging models to predict the L-value for mushroom samples in comparison to RGB-based models.

A multispectral vision system developed by Kleynen et al. (2005) included four wavelength bands in the visible/NIR range for sorting apples cv. Jonagold based on the presence of defects. They used interference filters centred at 450, 500, 750 and $800 \mathrm{~nm}$, but since the $500 \mathrm{~nm}$ spectral component did not give any significant information for discriminating between defects and sound tissue, finally this spectral component was not taken into account in computing the frequency distributions. The $450 \mathrm{~nm}$ spectral band provided significant information with which to identify slight surface defects like russet. The 750 and $800 \mathrm{~nm}$ bands, on the other hand, offered good contrast between the defect and the sound tissue and were well suited to detecting internal tissue damage like hail damage, bruises, and so forth. This system was recently used by Unay and Gosselin (2006) to study several thresholding and classification-based techniques for pixel-wise segmentation of multispectral images of cv. Jonagold apples using a multilayer perceptron-based method in order to detect surface defects. This work was later enhanced by extracting several features from defective skins with the aim of classifying the fruit in different categories (Unay et al., 2011).

Bennedsen and Peterson (2005) also developed a multispectral machine vision system with the aim of detecting surface defects on apples. The system operated on apples that were oriented with the stem/calyx axis perpendicular to the imaging camera. Images were acquired through two optical filters at 740 and $950 \mathrm{~nm}$. Due to the difference in the detecting ability of the two wavebands used, two training sets were constructed for each variety: one for $740 \mathrm{~nm}$ images and one for $950 \mathrm{~nm}$. In order to evaluate the overall performance of the system, the binary images resulting from the segmentations of the 740 and $950 \mathrm{~nm}$ images were combined. Apples of eight varieties were used to test the combined performance of the segmentation routines, with a success rate ranging from $78 \%$ to $92 \%$. Ariana et al. (2006) presented an integrated approach using multispectral imaging in reflectance and fluorescence modes to acquire images of three varieties of apples (Honeycrisp, Redcort and Red Delicious) to distinguish various defects on apples, including bitter pit, soft scald, black rot, decay and superficial scald. They acquired eighteen images from a combination of filters ranging from the visible region through the NIR region and from three different imaging modes (reflectance -R-, visible light-induced fluorescence and UV-induced fluorescence) for each apple as a basis for pixel-level classification into normal or damaged tissue. Seven band pass filters $(450,550,680,740,880,905$, and $940 \mathrm{~nm}$ peak transmittance) and a $710 \mathrm{~nm}$ long-pass filter were used in this system.

Blasco et al. (2007) developed a multispectral inspection system to detect and sort citrus fruits according to 11 different types of external defects by combining the information obtained from four image acquisition systems that are sensitive to NIR, visible, UV and fluorescence. Compared with the results obtained using only colour images, the multispectral system showed that the contribution of non-visible information increased the rate of success in fruit classification by about $78 \%$. This research was later enhanced in Blasco et al. (2009) to include some morphological features of defects, which raised the success rate up to $86 \%, 82 \%$ being the success rate when only an RGB camera was used. Single wavebands can be combined to create spectral indexes. Some of these indexes were studied by Lleó et al. (2011) to determine the ones that best fit the ripeness prediction of Richlady peaches, with two new indexes being proposed.

The detection of contaminants can be one of the applications of such multispectral systems. Kalkan et al. (2011) developed a two-dimensional local discriminant bases algorithm to discriminate between aflatoxin-contaminated and uncontaminated hazelnuts and red chili peppers flakes. The samples were screened with 12 different filters, some at 400-510 nm with $10 \mathrm{~nm}$ FWHM and others at 550 and $600 \mathrm{~nm}$ with 70 and $40 \mathrm{~nm}$ FWHM, respectively. The algorithm classified the flakes into aflatoxin-contaminated and uncontaminated classes with a $79.2 \%$ accuracy rate, so that the level of aflatoxin in the test set was decreased from $38.26 \mathrm{ppb}$ to 22.85 by removal of the ones that were classified as contaminated. The hazelnut kernels were independently subjected to two different classifications: first, on the detection of contamination and, second, on the detection of fungal infestation without considering their aflatoxin 
concentrations. A correct classification accuracy of $92.3 \%$ was achieved for classifying the hazelnuts as aflatoxin-contaminated $(>4 \mathrm{ppb})$ or not $(<4 \mathrm{ppb})$.

Internal quality can also be predicted using these systems. For instance, Peng and Lu (2005) presented a multispectral system with the objective of developing mathematical models to describe the relationship between fruit firmness and multispectral scattering profiles from apples. Scattering images were acquired from Red Delicious apples using two different multispectral imaging systems (a rotating filter and a multispectral imaging spectrograph) at wavelengths of $680 \mathrm{~nm}, 880 \mathrm{~nm}, 905 \mathrm{~nm}$ and $940 \mathrm{~nm}$ with a bandpass of $10 \mathrm{~nm}$. Each scattering image was reduced to a simple spatial scattering profile through radial averaging. In a different study aimed at estimating maturity, Lleó et al. (2009) used a multispectral imaging system to classify peaches into different levels of maturity at harvest and to compare this classification with reference measurements such as firmness or reflectance at $680 \mathrm{~nm}$ achieved with a visible spectrometer. The proposed system had three band-pass filters centred at $800 \mathrm{~nm}$ IR, $675 \mathrm{~nm}$ Red (R) and $450 \mathrm{~nm}$ Blue (B), with a bandwidth of $20 \mathrm{~nm}$. Two non-supervised classifications based on the Ward method were applied on the histograms extracted from the region of interest, i.e. the skin of the peach. The first classification considered the $\mathrm{R}$ channel image of each sample, while the second used the histograms of the R/IR images, which achieved better results (90\% agreement). The use of the R/IR ratio avoided the effect of fruit shape on light reflectance and thus improved the definition of multispectral maturity clusters. In contrast, the contribution of the B component in the classification was poor.

Beyond multispectral imaging, the use of hyperspectral sensors makes it possible to conduct a more sophisticated analysis of the scene by acquiring a set of images corresponding to particular wavelengths, normally in the visible and NIR part of the electromagnetic spectrum. The reduction in the price of hyperspectral systems, typically used for remote sensing and meteorology, allows them to be used in laboratories for food quality and they are an emerging and promising tool for food quality and safety control, as Gowen et al. (2007) stated in an earlier review of the use of this technology in food inspection. The acquired multidimensional spectral signature (spectrum) characterising a pixel can be used to analyse scenes like a standard colour camera but also to obtain information about internal compounds that can be related with the internal quality of the product.

These systems work with a large number of monochromatic images of the same scene at different wavelengths, thus enabling simultaneous analysis of the spatial and spectral information. The set of monochromatic images that are captured constitute a hyperspectral image. As they are made up of a large collection of images, hyperspectral images constitute a far more extensive source of information than that provided by a single monochromatic image or a conventional RGB image. The number of images depends on the spectral resolution of the system used and they are combined by forming a cube in which two dimensions are spatial (pixels) and the third is the spectrum of each pixel. Without adequate processing, such a large amount of data, despite being one of the main advantages of hyperspectral systems, can complicate the extraction of useful information, since much of the information obtained is redundant or, by nature, cannot be used to distinguish between regions with similar characteristics (Shaw \& Burke, 2003). The demanding industrial restrictions of working in realtime often make it necessary to reduce the dimensionality of the problem and to select the greatest amount of non-redundant information from the least number of wavelengths. Unsupervised methods such as principal component analysis or supervised ones such as linear discriminant analysis are commonly employed.

Another detail to bear in mind is that when raw hyperspectral images are analysed, it is the radiance of the scene rather than its reflectance that is being analysed. For these two reasons, when a hyperspectral image is acquired, first it is necessary to carry out the appropriate compensations in order to separate the reflectance of the scene from the radiance, and to apply techniques to reduce the amount of information obtained.

Some techniques for acquiring hyperspectral images even share technology with spectrometry, although the two techniques should not be confused. Hyperspectral imaging provides spectral and spatial information (what and where), while spectrometry provides information about spectral information captured at a particular spot on the sample. To know more about this technology, a good review about spectrometry was carried out by Nicolaï et al. (2007) or other literature can be consulted (Ozaki et al., 2006; Sun, 2009). Figure 1 shows a hyperspectral image of an orange with some external defects that are clear in particular wavelengths and practically invisible in others. 
Figure 1. Hyperspectral image decomposed on their monochromatic images showing an orange with some external defects.

This paper addresses the recent advances focused on the use of this technology for the quality assessment of fruits and vegetables. In the second section, different technologies for hyperspectral image acquisition are explained and discussed. Hyperspectral images generate a large amount of information that is important to reduce and analyse, being many works aimed at developing methods for this task. This works are described in the third section. Sometimes, the reduction of the data is aimed at selecting some important wavelengths in order to build multispectral systems that are easier to implement using a more standard technology. Works with this objective are revised in section four followed by the application of hyperspectral technology to the inspection of the external and internal quality of different species of fruits and vegetables. Finally, prospects of future trends and conclusions are given.

\section{Technologies for hyperspectral image acquisition}

The essential elements for constructing hyperspectral imaging systems include light sources, wavelength selection devices, and area detectors (Sun, 2010). Depending on the technology used, the selection of the wavelengths can be performed by dispersing the incident radiation into its individual wavelength or blocking the radiation in such a way that only the desired wavelength reaches the detector. The most frequently used are usually imaging spectrographs, liquid crystal tunable filters (LCTF) and, to a lesser extent, acousto-optic tunable filters (AOTF). Although these types are the most commonly used, there are also other kinds of equipment that have been developed for the acquisition of reflectance hyperspectral images (Kim et al., 2001).

\subsection{Liquid crystal tunable filters}

An LCTF is a solid-state instrument that uses electronically controlled liquid crystal cells to transmit light with a selectable wavelength, while excluding all others. The LCTF is based on Lyot filters, which are built from a series of optical stages, each consisting of a combination of a birefringent retarder (an optical property of a material that causes the polarisations of light to travel at different speeds) and a liquid crystal layer sandwiched between two parallel polarisers. As the incident linearly polarised light traverses the retarder, it is split into two light rays, i.e. the ordinary and the extraordinary rays, which have different optical paths though the retarder and emerge with a phase delay that is dependent on the wavelength. After transmission through the retarder, only those wavelengths that are in phase are transmitted by the polariser to the next filter stage (Hetch, 2003). To introduce tunability, a liquid crystal layer is used in each stage. Tunability is provided by the partial alignment of the liquid crystals along an applied electric field between the two polarisers. Tuning time for randomly accessing wavelengths depends on the liquid 
crystal material used and the number of stages in the filter. Typically, it takes tens of milliseconds to switch from one wavelength to another, which is far longer than the response time of the AOTF. While the position of the band-pass is actively tunable, its width is fixed and depends on the construction of the device. One advantage of the LCTF is a high rejection ratio for out-of-band transmission, which implies a high image quality. Another important advantage is that LCTFs can be manufactured with larger apertures than AOTFs. On the other hand, their major drawback is their longer tuning time relative to AOTFs, as previously commented.

A typical LCTF-based system includes the electronic module and the tunable filter. Gómez-Sanchis et al. (2008a) described the main elements, configuration and a spectral characterisation of an LCTF-based system for citrus inspection. The main characteristics included two liquid crystal filters, with spectral ranges of $400 \mathrm{~nm}$ to $720 \mathrm{~nm}$, and $650 \mathrm{~nm}$ to $1100 \mathrm{~nm}$ respectively. The combined use of both filters provided an extended working range from $400 \mathrm{~nm}$ to $1100 \mathrm{~nm}$. A uniform global efficiency of the system across all frequencies was achieved by varying the integration time for each band using a certified white reference. Images in those bands where the efficiency of the system was lower needed more acquisition time than others where the system was more efficient. In Peng and Lu (2006), an LCTF-based hyperspectral imaging system was developed for measuring fruit firmness of apples. Spectral images from Red Delicious and Golden Delicious apples were acquired from 650 to $1000 \mathrm{~nm}$ in increments of $10 \mathrm{~nm}$. Gómez-Sanchis et al. (2008a) studied the feasibility of an LCTF hyperspectral system for detecting decay in citrus fruits in the early stages of infection using halogen lighting instead of the traditional inspection using UV lighting. Two filters were used to achieve this: one that was sensitive to the visible $(460-720 \mathrm{~nm})$ and one that was sensitive to the NIR $(730-1020 \mathrm{~nm})$. Figure 2 shows a possible the arrangement for the simultaneous use of two LCTF filters (i.e. sensible to visible and NIR) with a single camera without alter the scene.

Figure 2. System for the acquisition of hyperspectral images based on two LCTF

\subsection{Acousto-optic tunable filters}

In recent years, technology based on AOTF has grown, thereby providing an alternative to LCTF and to imaging spectrographs (Vila et al., 2005), and its use is starting to be introduced for optimising agricultural and chemical processes (Bei et al., 2004). For instance, Jiménez et al. (2008) used an AOTF to obtain the spectrum of olive oil from inside a horizontal centrifugal decanter. This equipment allows instantaneous scanning of the oil flowing through the sensor. The imaging system was programmed to scan three spectra for each olive residue sample at a rate of $10 \mathrm{scans} / \mathrm{s}$ in the 1100 to $2250 \mathrm{~nm}$ range. Moreover, AOTF technology has also been used for the determination of physical features in food applications. Cayuela et al. (2009) described a portable AOTF-NIR spectrophotometer with a wide spectral range between 1100 and $2300 \mathrm{~nm}$, which was equipped with a reflectance post-dispersive optical configuration and an InGaAs detector was used for NIR prediction of fruit moisture content (MC), free acidity and oil content in intact olives. 
An AOTF is a solid-state device that works as an electronically tunable band-pass filter based on lightsound interactions in a crystal (Chang, 1976). It can isolate a single frequency of light from a broadband source in response to an applied acoustic field. The main components of an AOTF are a suitably oriented birefringent uniaxial crystal to which a piezoelectric transducer is bonded. The most common crystal for constructing an AOTF is Tellurium Dioxide $\left(\mathrm{TeO}_{2}\right)$. The application of a radio frequency (RF) signal to the transducer produces an acoustic wave that propagates inside the crystal. The acoustic waves change the refractive index of the crystal by compressing and relaxing the crystal lattice. The changes in the refractive index make the crystal act like a transmission diffraction grating. Unlike a classical diffraction grating, the AOTF only diffracts one specific wavelength of light, so that it acts more like a band-pass filter with a narrow bandwidth than a diffraction grating. The diffracted light is divided into two beams with orthogonal polarisations, i.e. horizontally polarised and vertically polarised. The undiffracted beam and the undesired diffracted beam (e.g. vertically polarised beam) are blocked by a beam stop. The wavelength of light selected is a function of the frequency of the RF applied to the crystal. Therefore, the wavelength of the diffracted beam is controlled by changing the frequency of the RF source (Vila-Francés et al., 2011). Furthermore, the bandwidth and the intensity of the filtered light can also be adjusted by controlling the RF source. In addition, if multiple RF frequencies are launched into the crystal, then combinations of frequencies can be diffracted simultaneously, which makes it more flexible than LCTFs, which generate only a single band-pass at a time. Since AOTF is an advanced electronically tunable filter, it includes important features similar to those to be found in LCTF, such as accessibility to random wavelengths, flexible controllability, high spectral resolution, fast wavelength switching, wide spectral range, narrow bandwidth, and a relatively large optical aperture. AOTF technology presents, as its main advantage, a tuning speed that is higher than that obtained with LCTF technology, typically in tens of microseconds, owing to the fact that it is only limited by the speed of the sound propagation in the crystal. Furthermore, AOTF presents broader wavelength ranges (Vila-Francés et al., 2010). On the other hand, AOTFs have smaller apertures compared with LCTFs and, in addition, imaging quality is comparatively poor.

\subsection{Imaging spectrographs}

An imaging spectrograph is an optical device that is capable of dispersing incident broadband light into different wavelengths instantaneously on an area detector (e.g. a CCD detector). Wavelength dispersion is carried out using a prism. The imaging spectrograph generally operates in a line-scanning mode, i.e. the object is scanned line-by-line as the entire field of view is acquired. The light from a scanning line is dispersed into different wavelengths and they are projected onto the area detector, creating a special twodimensional image: one dimension represents spatial information and the other the spectral dimension. Each vertical line along the spectral axis of the 2D area detector forms a continuous spectrum from a fixed spatial point on the object surface. The object must be moved stepwise under the acquisition system by means of a stepper table while at each step a line is scanned, as in a push broom scanner. Thus, a full spectral image can be obtained. One advantage of the imaging spectrograph is its high spectral resolution. On the other hand, the major drawback is the need to move the object with respect to the spectrograph. Therefore, it is not possible to acquire an entire image without properly synchronising the image acquisition with the movement of the object. This fact makes hyperspectral image acquisition difficult and thus makes it necessary to use complex calibration techniques in order to achieve quality hyperspectral images (Sun, 2010).

This technology is cited in the scientific literature more frequently than LCTF and AOTF technologies for agricultural applications probably because of its higher spectral range and resolution. Some examples where this technology is well described include ElMasry et al. (2008), where a hyperspectral imaging system based on a spectrograph was used in the spectral region between 400 and $1000 \mathrm{~nm}$ for early detection of bruises on different background colours of apples cv. McIntosh. Three effective wavelengths in the NIR region $(750,820,960 \mathrm{~nm})$ were found that could potentially be implemented in multispectral imaging systems for the detection of bruises on apples. In the study conducted by Al-Mallahi et al. (2008), a hyperspectral imaging system was used to distinguish potato tubers from soil clods. An imaging spectrograph was employed to take hyperspectral images of 60 wavebands in the 321-1044 nm range. In order to improve success rates, hyperspectral images were taken and analysed to find the most relevant wavebands to perform an optimum discrimination. This paper highlights the usefulness of employing an imaging spectrograph to discriminate between tubers and clods and shows the significance of adding a waveband from the NIR to accomplish a high success rate of discrimination under any moisture conditions. Such systems have also been successfully used for the evaluation of physical properties in food science. For instance, Polder et al. (2003) used an imaging spectrograph (393-710 nm) to estimate lycopene and chlorophyll contents, which play a role in the ripening of tomatoes. They also used an imaging spectrograph to measure the concentration of the main constitutive compounds in tomatoes 
(including lycopene, lutein, $\beta$-carotene, chlorophyll-a and chlorophyll-b) at different stages of ripening (Polder et al., 2004). Spectral images from 396 to $736 \mathrm{~nm}$ with a resolution of $1.3 \mathrm{~nm}$ were recorded and used to determine the presence of these compounds. Figure 3 shows a possible arrangement of a hyperspectral system based on image spectrograph. The sample has to be placed over a conveyor belt or a similar mechanism that displaces it under the camera along the image is acquired. A possible alternative us the use of a mirror scan that is a device that captures the image reflected on a moving mirror.

Figure 3. A possible arrangement for hyperspectral imaging spectrograph

\section{Most commonly used statistical techniques}

Having a large number of bands is of great interest but also increases the complexity of the analysis of the information. The statistical problems that arise when multicollinearity in a set of variables to be analysed is present makes the selection of important wavelengths or classification of pixels for hyperspectral image segmentation difficult (Mather, 1998). This section analyses the most widely used statistical techniques found in the scientific literature for this purpose: Principal Component Analysis (PCA), Partial Least Squares (PLS), Linear Discriminant Analysis (LDA) and Artificial Neural Networks (ANN). It should be noted however, that most of these techniques are focused just on the spectral raw data without including information that is spatially adjacent in the images; hyperspectral data are not treated as images but as simple values stored in a spectral array. However, spatial information can be included afterwards in the images obtained from the most important sources of variability (score images).

\subsection{Principal component analysis}

PCA is one of the most popular multivariate statistical techniques, commonly used to reduce the dimensionality of data and to solve the multicollinearity problem. This method simplifies the task of obtaining an overview of all the information in the data set, because it is an unsupervised projection method which summarises data by forming new variables as uncorrelated and linear combinations of the original variables. Normally, a few of these new variables, called principal components (PCs), should explain most of the common variations in all the data. Therefore, by only considering several PCs, high dimensional data can be reduced to a lower dimensionality with a minimal loss of information. A drawback of PCA is that it does not guarantee the class separability of data due to its unsupervised nature related to the fact that PCA summarises the variance in the data, which may not be related to the segregation of the classes (Jollife, 1986). This technique is widely used in hyperspectral imaging, as it is considered a powerful and robust tool for obtaining an overview of such complex data and for reducing the large dimension of the data provided by the hyperspectral images. For example, Mehl et al. (2004) used this technique on hyperspectral images $(628-900 \mathrm{~nm})$ to detect different defects and contaminations on the surface of apples. Differences in spectral responses were analysed using PCA and second difference methods for sorting wholesome and contaminated apples. The study showed that both methods gave very similar results for the detection of disease, fungal contamination, bruises and soil contamination on apples. The main differences were related to the computing time. While PCA is more 
complex to use and requires more data processing time, the asymmetric second difference method only required three wavelengths and much less computation time to process the images.

The PCA technique has been widely applied for data reduction. For instance, Xing et al. (2005) used it to reduce data from a hyperspectral imaging system $(400-1000 \mathrm{~nm})$ for detecting bruises on 'Golden Delicious' apples. Four wavebands centred at 558, 678, 728 and $892 \mathrm{~nm}$ were selected for later use in a multispectral imaging test using PCA. Then, if there was one bruised area recognised on the apple surface using thresholding, the fruit was classified as bruised. The classification results indicated that about $93 \%$ of the non-bruised apples were recognised as sound, and an accuracy of about $86 \%$ was achieved in bruised apples. Later, Xing et al. (2007a) used PCA in the same spectral region to reduce the number of bands for separating stem-end/calyx regions from true bruises on apples cv. Golden Delicious and cv. Jonagold. They found four (558, 678, 728 and $892 \mathrm{~nm})$ and six $(571,608,671,709$, 798 and $867 \mathrm{~nm})$ effective wavebands for identifying the stem-end/calyx, respectively, in the two varieties. Then, stemend/calyx regions were distinguished from other skin defects by analysing the contour features of the first PC score images. In the investigated samples, none of the sound tissue was misclassified as a stem-end or calyx region for either of the cultivars, and all of the cases of stem-end/calyx present in the images were correctly recognised for the Golden Delicious apples and $98.33 \%$ for Jonagold apples. Finally, less than $3 \%$ of bruises were misclassified as stem-end/calyx regions in the two cultivars. With the same configuration and for the same purposes, Xing et al. (2007b) used the chemometric tool PCA to extract and summarise the spectral information from the hyperspectral images. PCA was then carried out on the same four effective wavebands as before. Later, PC2 and PC3 score images were combined to construct virtual images, and finally the 'moments' thresholding method was used to process those virtual images, this tool being found to be suitable for this application.

In the work by Gowen et al. (2008), the PCA method was used on a hyperspectral imaging system in the spectral region between 400 and $1000 \mathrm{~nm}$ with a resolution of $5 \mathrm{~nm}$ in order to detect bruise damage on white mushrooms. Two data reduction methods were investigated: in the first one, PCA was applied to the hypercube of each sample and the second PC (PC2) score image was used to identify bruise-damaged regions on the mushroom surface. In the second one, PCA was applied to a data set consisting of average spectra from normal regions and bruise-damaged tissue. Better results were obtained with the second method when applied to a set of independent mushroom samples. The score image was also used prior to several classification algorithms to detect bruises on pears cv. Crystal. Zhao et al. (2010) applied PCA to the spectral region ranging from 526 to $824 \mathrm{~nm}$ in order to get the best score image representing the bruised region. It was found that the bruised region could be clearly identified from the PC2 score image.

\subsection{Partial least squares}

PLS regression is an unsupervised statistical method used when not only a data array coming from $X$ data is available, but also a Y array that we want to predict from our X data. The aim of PLS analysis is to find a latent variables linear regression model by projecting the $\mathrm{X}$ variables and the $\mathrm{Y}$ variables into a new latent space, where the covariance between these latent variables is maximized. In other words, a PLS model tries to find the latent multidimensional direction in the $\mathrm{X}$ space that explains the direction of the maximum multidimensional covariance in the Y space. The PLS algorithm determines a set of orthogonal projection axes, called PLS-weights. Typically, most of the variance could be captured with the first few latent variables, while the remaining latent variables described random noise or linear dependencies between the dependent variables and the independent variables. Moreover, PLS analysis is related to PCA. Both techniques are used to convert a set of highly correlated variables into a set of independent latent variables ( $\mathrm{t}$ scores) by using linear transformations and can be employed as dimension reduction techniques. Due to all these benefits, PLS models are widely used in hyperspectral imaging to extract and summarise spectral information from hyperspectral images, to reduce the high dimensionality of the spectral data and to overcome the problem of multicollinearity, when we want to infer some Y property from the former (hyperspectral images) (Vinzi et al., 2010).

This technique was used by ElMasry et al. (2007) to analyse hyperspectral images in the visible and NIR $(400-1000 \mathrm{~nm})$ regions for the non-destructive determination of MC, SSC and acidity (expressed as pH) quality attributes in strawberry. PLS models were developed between the average reflectance spectra and the measured quality parameters in order to predict quality parameters. Furthermore, multiple linear regression (MLR) models were established using only the optimal wavelengths to predict the quality attributes. Moreover, for classifying strawberry based on the stage of ripeness, a texture analysis was conducted on the images based on grey-level co-occurrence matrix (GLCM) parameters. The MLR models demonstrated higher prediction performance than PLS models, although only a few wavelengths 
were utilised. In order to study ripening in tomatoes, Polder et al. (2004) analysed concentrations of different compounds using HPLC and by analysing spectral images using PLS regression at the pixel level and at the tomato level. PLS regression models at the tomato level were constructed either by averaging the prediction of all the pixels or by training the model on the mean of the spectra of all the pixels (i.e. only one spectrum is used per tomato). Although regression on complete tomatoes gave a smaller error than regression on individual pixels, pixel-based regression made it possible to construct concentration images of tomatoes with non-uniform ripening.

Partial least squares discriminant analysis (PLS-DA) is a supervised variant of PLS regression where the independent variable is a categorical one expressing the class membership of the samples. The usual PLS Discriminant Analysis was proposed to overcome the multicollinearity problem of LDA and it can be defined as a straightforward extension of the PLS regression (Sjöström et al., 1986). Using a PLS-DA calibration model, Nicolaï et al. (2006) discriminated between pixels of sound apple skin and bitter pit lesions. Leave-one-out cross validation was used to determine the number of latent variables based on minimisation of the root mean squared error of cross validation prior to segmentation. The number of latent variables in the PLS calibration was two and the system was able to identify bitter pit lesions, even when they were not visible to the naked eye, such as just after harvest. In the work by Xing et al. (2007b), a PLS-DA model was also built to extract and summarise spectral information. The pixels from the bruised regions were assigned a value of zero and the pixels from the sound tissue were given a value of one. These values were then used as dependent variables and the normalised reflectance spectra were taken as the independent variables in the PLS-DA model. Similar to the method used in the PCA procedure shown in section 3.1, the loadings plots of PLS latent variables were used to determine the effective wavebands for discrimination. The PLS-DA model was later rebuilt with the same four effective bands and by arranging the prediction values for each pixel on an apple. The authors concluded that PLSDA does not need complex image processing of the virtual images, but it does need a good training data set to be able to build a model prior to predicting new samples (as well as any statistical model, although this is even more evident in high dimensional data arrays and supervised models). Moreover, poorer results for the identification of stem-end/calyx were achieved than with PCA score images. Menesatti et al. (2009) studied the spectral region between 1000 and $1700 \mathrm{~nm}$ using PLS-DA with the aim of developing an objective method to assess the starch index in apples cv. Golden Delicious that avoided the need for chemical methods, although it is still destructive. They applied PLS-DA to hyperspectral NIR images to classify single pixels into starch or starch-free classes, using their NIR reflectance spectrum. The starch classification of each apple was based on the percentage of classified starch-free areas over the total (starch-free and starch) classified pixels. The potential of hyperspectral imaging (445-945 nm) to discriminate between casing soil, enzymatic browning and undamaged tissue on mushroom surfaces was investigated by Taghizadeh et al. (2011). Damage-free mushrooms, mushrooms artificially smeared with casing soil and mechanically damaged mushrooms, resulting in enzymatic browning, were tested. PLSDA models were developed to classify mushroom tissue as one of the three classes investigated using pixel spectra from each class. Prediction maps were obtained by applying the developed models to the hyperspectral images of candidate mushrooms. Percentages of pixels classified into each class were also calculated for the mushrooms studied in the calibration set. Results obtained showed that the models performed satisfactorily in discriminating between the three classes studied. Model validation was performed using three different test sets of mushrooms obtained from a commercial producer. It was found that the PLS-DA models that were developed were capable of satisfactorily identifying undamaged regions, casing soil and enzymatically damaged areas on mushrooms from the validation sets.

\subsection{Linear discriminant analysis}

Discriminant analysis is a statistical technique for classifying objects into mutually exclusive groups (classes) based on a set of measurable features of the objects, which in the case of hyperspectral images are normally spectral features. This supervised method is focused on maximizing the ratio of the variance between groups and variance within groups (Jobson, 1992). The class membership of a sample can be predicted by calculating the distance to the centroid of each class in the transformed space and then assigning the sample to the class with the smallest distance to it. LDA has no free parameters to be tuned and the extracted features are potentially interpretable under linearity assumptions. This technique is related to ANOVA (analysis of variance) and regression analysis, which also attempt to express one dependent variable as a linear combination of other features or measurements (Fisher, 1936; McLachlan, 2004). Furthermore, it is also closely related to PC when dealing with multidimensional data arrays, since LDA needs the $\mathrm{X}$ variables to be independent and normally distributed, which can be easily performed by PCA feature extraction (working on the scores or latent variables obtained from PCA. However, this technique does not take into account any differences in class (Martinez \& Kak, 2004). These capabilities have led to its extensive use and practical exploitation in many fields of application, such as hyperspectral 
imaging, which involves a large number of features. Polder et al. (2002) compared a hyperspectral system to a standard RGB one for measuring the stages of ripeness of tomatoes. The spectrograph had a spectral range of 396 to $736 \mathrm{~nm}$ with a spectral resolution of $1.3 \mathrm{~nm}$, which enabled it to obtain spectral images with 257 bands. LDA was performed on both hyperspectral and RGB images, as well for classifying pixels in five different classes of ripeness. The spectral bands of each pixel were used as independent variables in the analysis of the images after reducing the dimensionality of the hyperspectral images to three bands to enable the comparison of both systems. The classification of tomatoes was performed by a simple majority vote regarding individual pixel classification, the spectral images being more discriminating than standard RGB images. On the other hand, Al-Mallahi et al. (2008) used linear discriminant analysis to distinguish potato tubers from soil clods. Images were taken under wet and dry conditions and segmentation was performed using linear discriminant analysis, the result being pixelsegmented images in which each pixel was classified as tuber or clod, and when the proportion of the tuber pixels exceeded a certain majority percentage, the entire object was classified as a tuber, otherwise as a clod. In order to improve the success rates obtained with RGB images, five effective bands from hyperspectral images were analysed. The discriminant functions from LDA were able to separate the pixels and classify the objects as tuber or clod under wet and dry conditions with higher rates than with just colour information. In this case, the hyperspectral images analysed using LDA also offered better results than the traditional RGB systems. Gómez-Sanchis et al. (2008b) used LDA and classification and regression trees (CART) to segment multispectral images of rotten and sound mandarins. Altogether 20 spectral features corresponding to 20 monochromatic images were used as independent variables in the model.

In order to sort citrus fruits by identifying the most common defects, Blasco et al. (2007) developed a multispectral inspection system which combined the information obtained from four image acquisition systems that were sensitive to NIR, visible, UV and fluorescence. Segmentation of the four images was carried out using an unsupervised region-growing process. LDA was used to segment visible images, where the colour coordinates of pixels were used as independent variables. A comparison of the results from colour images with those obtained from the multispectral system showed that the contribution of non-visible information increased the rate of successful fruit classification, especially in the case of some of the most dangerous defects.

Gowen et al. (2009a) used LDA to analyse hyperspectral images for the early detection of freeze damage in white button mushrooms. For each image, mean reflectance spectra from the central part were obtained and PCA was applied to the calibration set of data in order to concentrate that spectral information into the two first PC scores. LDA was then applied in order to estimate a boundary to separate the clusters of undamaged and freeze-damaged spectra. Using this method, freeze-damaged mushrooms could be correctly classified with an accuracy $>95 \%$ after 45 minutes thawing, even when freeze-thaw damage was not visibly evident. The reasonably low misclassification rates obtained for classification of undamaged mushrooms and mushrooms just after thawing highlights the high potential of hyperspectral imaging combined with PCA to reduce the original data dimensional space and LDA for the early identification of mushrooms subjected to freeze damage.

\subsection{Artificial neural networks}

An ANN is a non-linear statistical data-modelling tool that attempts to mimic the fault-tolerance and capacity to learn of biological neural systems by modelling the low-level structure of the brain. An ANN consists of an interconnected group of artificial neurons that works like a parallel system capable of resolving paradigms that linear computing cannot. In most cases, an ANN is an adaptive system that can change and adjust its knowledge by adjusting its parameters according to the samples of data that are presented in order to solve the problem at hand. This is called the learning phase. They are usually used to model complex relationships between inputs and outputs or to find patterns in data. The most popular ANN is the multilayer perceptron (MLP), which is a feedforward ANN model that maps sets of input data onto a set of appropriate outputs, and consists of multiple layers of nodes (neurons) on a directed graph that is fully connected from one layer to the next. MLP can employ a large variety of learning techniques, the most popular being backpropagation. The backpropagation algorithm is a supervised learning method based on gradient descent in error which propagates classification errors back through the network and uses these errors to update parameters (Shih, 2009). ANN is a commonly used pattern recognition tool in hyperspectral image processing because of the fact that it is capable of handling a large amount of heterogeneous data with considerable flexibility and due to its non-linear property (Plaza et al., 2009). Furthermore, ANN have several advantages over conventional pattern recognition methods. Firstly, they can learn the intrinsic relationship by example. Secondly, they are more fault-tolerant than conventional 
computational methods and, finally, in some applications, ANN are preferred over statistical pattern recognition because they require less domain-related knowledge of a specific application (EgmontPetersen, 2002).

Because of their flexibility and the possibility of working with unstructured and complex data like that obtained from biological products, ANN have been applied in almost every aspect of food science, and it is a useful tool for performing food safety and quality analyses (Huang et al., 2007). For instance, Qin and $\mathrm{Lu}$ (2005) developed a feedforward backpropagation ANN classifier to sort cherries with and without pits using a transmission hyperspectral imaging system. The images were acquired in four orientations before and after pits were removed. Additional cherries were bruised and then subjected to two different postbruising treatments in order to study the bruising effect on pit detection. Single spectra obtained from a specified spatial location of the image and selected regions of interest (ROIs), both covering the spectral region between $692 \mathrm{~nm}$ and $856 \mathrm{~nm}$, were compared as inputs for the ANN. To reduce the data dimensionality, PCA was applied to the ROI data and only score spectra of the first PC were used as inputs of the ANN. A sample was classified as having a pit when the output value was greater than the threshold value (0.5), and as having no pit otherwise. ROIs resulted in a $3.5 \%$ error in incorrect classification of cherries with pits and a $3.1 \%$ error for cherries without pits, which are less than half of those from single spectra. Results showed that fruit size and defects had an important effect on pit detection but the effect of fruit orientation or colour on ANN classifications was negligible. A combination of PCA and ANN was also used by Bennedsen et al. (2007) to detect surface defects on apples cv. Golden Delicious. The images were arranged as data sets in which each individual column was considered a sample, and the pixel values were taken as variables. By using PCA, the columns of pixels were replaced by columns of PCs, where each column was assigned a value of 'zero' if it did not represent a defect and 'one' if it did. This matrix was used to train the subsequent neural networks. There are four network categories: two sets based on $740 \mathrm{~nm}$ images and two based on $950 \mathrm{~nm}$ images. Each set consisted of two categories based on the arrangement of the images: 'vertical' and 'horizontal'. The 'vertical' position is how the images are seen by the camera, where the stem/calyx axis was vertical. The network outputs for each of two wavebands were then divided by two and added together to produce a new image. If there was an intersection of the two networks with a positive value, the defect was considered to have been detected. The overall performance of the system was evaluated and found to be non-suitable by itself for practical implementation due to its low detection rate.

Unay and Gosselin (2006) applied several thresholding and classification-based techniques for pixel-wise segmentation of surface defects on apples cv. Jonagold. In this work, they used 18 segmentation techniques: three global thresholding techniques (Entropy, Isodata and Otsu), one local thresholding technique (Niblack's method), three unsupervised classifiers (k-means, competitive neural networks and self-organising feature maps) and 11 supervised classifiers in which MLP was included. Results showed that among many classification and thresholding-based methods, MLP was the most promising for segmenting surface defects in high-speed machine vision-based apple inspection systems. For different cultivars of apples, Ariana et al. (2006) combined multispectral imaging in reflectance and fluorescence imaging modes in one integrated approach in order to acquire images with which to distinguish several defects including bitter pit, soft scald, black rot, decay and superficial scald. Backpropagation ANN classification models were developed for two classification schemes (binary and multi-class). Seven classification models for each variety were built based on imaging mode combinations, the integrated imaging model of reflectance and fluorescence (FUV+R) being more effective on cv. Honeycrisp apples, whereas single imaging models of visible and NIR reflectance or UV-induced fluorescence were effective on cv. Redcort and cv. Red Delicious.

Neural networks were also used by ElMasry et al. (2009) by means of feedforward backpropagation ANN models, which were developed to classify the apples into injured and normal classes, and to detect changes in firmness due to chilling injury. Five optimal wavelengths (717, 751, 875, 960 and $980 \mathrm{~nm}$ ) were selected by an ANN model based on the maximum weight assigned to the input nodes. The ANN model developed to predict the firmness of the apples had the spectral responses of an apple at the selected optimal wavelengths and used averaging to obtain the spectral value of all pixels as input nodes and the apple firmness value as output. Finally, to differentiate damaged apples from normal ones, the output layer of the ANN model developed was then modified so as to have two nodes: normal and injured classes. Table 1 summarises important works that use the described statistical techniques for the processing of hyperspectral images. 
Table 1. Studies related to statistical techniques applied to hyperspectral image processing

\begin{tabular}{|c|c|c|c|}
\hline Reference & Fruit & $\begin{array}{c}\text { Statistical } \\
\text { techniques }\end{array}$ & Application \\
\hline Chichester et al. (2003) & Tomatoes & ICA & $\begin{array}{l}\text { Lycopene and chlorophyll contents } \\
\text { to estimate ripeness }\end{array}$ \\
\hline Mehl et al. (2004) & $\begin{array}{l}\text { Red Delicious, } \\
\text { Golden Delicious, } \\
\text { Gala, and Fuji apples }\end{array}$ & PCA & $\begin{array}{l}\text { Detection of disease, fungal } \\
\text { contamination, bruises and soil } \\
\text { contamination }\end{array}$ \\
\hline Xing et al. (2005) & $\begin{array}{l}\text { Golden Delicious } \\
\text { apples }\end{array}$ & PCA & $\begin{array}{l}\text { Reduction in the number of } \\
\text { hyperspectral bands for bruise } \\
\text { detection }\end{array}$ \\
\hline Xing et al. (2007a) & $\begin{array}{l}\text { Golden Delicious } \\
\text { and Jonagold apples }\end{array}$ & PCA & $\begin{array}{l}\text { Reduction in the number of } \\
\text { hyperspectral bands for bruise } \\
\text { detection }\end{array}$ \\
\hline Xing et al. (2007b) & $\begin{array}{l}\text { Golden Delicious } \\
\text { and Jonagold apples }\end{array}$ & PCA \& PLS & Stem-end/Calyx-end detection \\
\hline Gowen et al. (2008) & White mushrooms & PCA & Freeze damage \\
\hline Zhao et al. (2010) & Crystal pears & PCA & Bruise detection \\
\hline ElMasry et al. (2007) & Strawberry & PLS & $\begin{array}{l}\text { MC, SSC, and acidity (expressed as } \\
\text { pH) quality attributes }\end{array}$ \\
\hline Polder et al. (2004) & Tomatoes & PLS & $\begin{array}{l}\text { Constitutive compounds at different } \\
\text { ripening stages }\end{array}$ \\
\hline Nicolaï et al. (2006) & Apples & PLS & Identification of bitter pit lesions \\
\hline Menesatti et al. (2009) & $\begin{array}{l}\text { Golden Delicious } \\
\text { apples }\end{array}$ & PLS & Assess starch index \\
\hline Polder et al. (2002) & Tomatoes & LDA & $\begin{array}{l}\text { Compare hyperspectral system to } \\
\text { standard RGB for ripeness stages }\end{array}$ \\
\hline Al-Mallahi et al. (2008) & Potatoes & LDA & $\begin{array}{l}\text { Distinguish potato tubers from soil } \\
\text { clods }\end{array}$ \\
\hline $\begin{array}{l}\text { Gómez-Sanchis et al. } \\
\text { (2008b) }\end{array}$ & $\begin{array}{l}\text { Clemenules } \\
\text { mandarins }\end{array}$ & LDA & Decay \\
\hline Blasco et al. (2007) & Citrus fruits & LDA & Segmentation of visible images \\
\hline Gowen et al. (2009) & White mushrooms & LDA & Early detection of freeze damage \\
\hline Qin and Lu (2005) & $\begin{array}{l}\text { Montmorency tart } \\
\text { cherries }\end{array}$ & ANN & Sort cherries with and without pits \\
\hline Bennedsen et al. (2007) & $\begin{array}{l}\text { Golden Delicious } \\
\text { apples }\end{array}$ & ANN & Identification of surface defects \\
\hline Unay and Gosselin (2006) & Jonagold apples & ANN & $\begin{array}{l}\text { Pixel-wise segmentation of surface } \\
\text { defects }\end{array}$ \\
\hline Ariana et al. (2006) & $\begin{array}{l}\text { Honeycrisp, Redcort } \\
\text { and Red Delicious } \\
\text { apples }\end{array}$ & ANN & $\begin{array}{l}\text { Distinguish bitter pit, soft scald, } \\
\text { black rot, decay and superficial } \\
\text { scald defects }\end{array}$ \\
\hline ElMasry et al. (2009) & Red Delicious apples & ANN & Predict the firmness of the apples \\
\hline
\end{tabular}

\section{Dimensionality reduction and selection of spectral features}

A typical hyperspectral image is composed of dozens of correlative monochromatic images. With a spectral resolution of about $5 \mathrm{~nm}$, a system working between 400 and $1000 \mathrm{~nm}$ could acquire about 120 images (features), which normally contain redundant information or may exhibit a high degree of correlation. In addition, the computational complexity of the classifier can become excessive for high dimensional data sets. The optimal reduction in dimensionality allows the performance system to be optimised in terms of computational performance and simplicity. These problems are commonly alleviated by using techniques that retain most of the original information in fewer bands. These include several techniques that aim to conserve the greatest amount of variability and the most significant information contained in the hyperspectral image (Du and Sun, 2006). Guyon and Elisseeff (2003) summarised the main benefits of variable selection as improving the inference performance, providing faster and cost effective predictors, and better understanding of the underlying process that generates the data. Methods for reducing the dimensionality can be divided into feature selection and feature extraction. Feature selection approaches try to find a subset of the original variables, i.e. frequency bands in the case of hyperspectral images. In some cases, data analysis such as regression or classification can be performed in the reduced space more accurately than in the original space. Feature extraction, on the other hand, transforms the data in the high-dimensional space into a space with fewer dimensions. The data transformation may be linear, as in PCA, but many non-linear dimensionality reduction techniques, such as ANN, also exist (Lee and Verleysen, 2007). 
Many of the works related with the inspection of fruit and vegetables that use hyperspectral systems to acquire the images are aimed at reducing hyperspectral to multispectral information in order to develop systems capable of obtaining similar results using simpler technology or that could be used for in-line applications. Xing and Baerdemaeker (2005) used a hyperspectral imaging system based on a spectrograph (400-1000 $\mathrm{nm}$ ) to obtain optimal waveband images for detecting bruises on apples cv. Jonagold, and capable of separating them from the stem and calyx. PCA was used to reduce the high dimension of the data provided by the spectral reflectance image. In accordance with PCA loading plots, six wavebands centred at 571, 608, 671, 709, 798 and $867 \mathrm{~nm}$ were selected. The contour plots for the PC1 scores were useful to distinguish between sound apple skin, bruised apple and apples with calyx and stem-end. Using just these wavelengths, no misclassifications were observed for the bruise class. Lefcout et al. (2006) proposed a robust method for selecting one or two wavelengths from hyperspectral data with the aim of detecting faeces in Golden and Red Delicious apples, using reflectance and fluorescence images. The apples were inspected from 452 to $729 \mathrm{~nm}$ and from 465 to $900 \mathrm{~nm}$. The segmentation of the images was accomplished by applying a binary threshold to images of a single wavelength and to images constructed using ratios or differences between images at two different wavelengths. For reflectance imaging, maximum detection rates for spots of 1:20-dilution faeces were $100 \%$ and $62.5 \%$ using R816-R697 and R784-R738, respectively. For fluorescence imaging, maximum detection rates for spots of 1:200-dilution faeces were $97.9 \%$ and 58.3\% using F665/F602 and F647/F482, respectively. In all cases, the results reached a success rate of $100 \%$ if the dilution was increased. Maximum detection rates for Red Delicious apples required the use of a Prewitt edge-detection filter. PCA was also used by Liu et al. $(2005,2006)$ to obtain spectral features for the detection of chilling injury in cucumbers imaged using a hyperspectral system based on a spectrograph with a spectral range of 447-951 nm. A large spectral difference between good, smooth skins and chilling-injured skins occurred in the 700-850 nm visible/NIR regions. Hence, both simple band ratio algorithms and PCA were used in an attempt to discriminate ROI spectra of good cucumber skins from those injured by chilling. These methods were applied to the mean reflectance spectra of cucumber ROI areas. The PCA classification model was established using two classes, 'good' and 'injury', based on SIMCA (Soft Independent Modelling of Class Analogy, Sjöström et al., 1986) of PCA with a Mahalanobis distance and a residual spectral measurement. On the other hand, a simple band ratio algorithm $(\mathrm{Q}=\mathrm{R} 811 / \mathrm{R} 756)$ was also used. Results revealed that both the dual-band ratio algorithm $(\mathrm{Q}=\mathrm{R} 811 / \mathrm{R} 756)$ and the PCA model from a narrow spectral region of 733-848 nm can detect chilling-injured skins of cucumbers within 3-7 days' room temperature (RT) storage with a success rate of over $90 \%$.

Gómez-Sanchis et al. (2008b) analysed the feasibility of detecting rottenness in citrus fruits caused by Penicillium digitatum in the early stages of infection using a hyperspectral computer vision system. The aim was to find a reduced set of optimally selected bands with which to develop a system that could replace the current ones based on dangerous UV-induced fluorescence lighting. The hyperspectral vision system used two LCTF to achieve the goal: one sensitive to the visible $(460-720 \mathrm{~nm})$ and one sensitive to NIR (730-1020 nm). Four feature-selection methods were evaluated in order to select the most discriminant bands for distinguishing between early fungal damage and sound skin, such as correlation analysis, mutual information, stepwise multivariate regression and genetic algorithms based on LDA. The minimum number of bands required to optimise successful classification was estimated to be 20 , which was obtained with the GALDA method.

As already mentioned, PLS is one of the most commonly used methods for this purpose. ElMasry et al. (2008) determined some important wavelengths for detecting bruises in apples cv. McIntosh using this technique on hyperspectral images. A hyperspectral imaging system based on a spectrograph was used to acquire images between 400 and $1000 \mathrm{~nm}$. PLS and stepwise discriminant analysis were used to reduce data dimensionality and to select the effective wavelengths. Three wavelengths in the NIR region (750, $820,960 \mathrm{~nm}$ ) were found that could potentially be implemented in multispectral imaging systems for the detection of bruises in this cultivar of apples. The images at the selected wavelengths were averaged, thereby creating a new image that was the basis for bruise area identification using a multilevel adaptive thresholding method. The results indicated that the bruised apples could be successfully distinguished from the sound apples from one hour after bruising. Using PLSR, Gowen et al. (2009b) studied spectral bands related with water in order to investigate the spectral behaviour of white mushrooms under different levels of mechanical vibration, using a hyperspectral imaging system based on a spectrograph and operating in the NIR wavelength of 950-1700 nm. Candidate water matrix coefficients of mushrooms under perturbation by physical vibration may be associated, respectively, with strongly and weakly bound water in the mushroom matrix. PLSR models were built for the prediction of vibration time, using mean absorbance spectra as inputs. Changes in sample spectra arising from perturbation were examined by 
observation of PLSR coefficients. Candidate water matrix coordinates were proposed at 950, 1174, 1398, 1433, 1454, 1496 and $1510 \mathrm{~nm}$. Absorbance spectra at these wavelengths suggested that there was a decrease in absorption at wavebands associated with strongly bound water accompanied by an increment in absorption at wavelengths associated with weakly bound water as vibration time increased, indicating mobility of water on the mushroom surface after perturbation.

A different approach was taken by Ariana and Lu (2010), who used hyperspectral imaging under the transmittance mode to select important wavebands that can be used in a further development of an in-line inspection system to detect internal defects in pickling cucumbers and whole pickles. Hyperspectral images were acquired from normal and defective cucumbers and whole pickles using a hyperspectral reflectance system $(400-740 \mathrm{~nm})$ and a transmittance imaging system $(740-1000 \mathrm{~nm})$. A total of four subsets of wavebands were determined by a branch and bound algorithm combined with the k-nearest neighbour classifier. The highest classification accuracies of $94.7 \%$ and $82.9 \%$ were achieved using the optimal four-waveband sets of 745, 805, 965, and $985 \mathrm{~nm}$ at $20 \mathrm{~nm}$ spectral resolution for cucumbers and of $745,765,885$, and $965 \mathrm{~nm}$ at $40 \mathrm{~nm}$ spectral resolution for whole pickles, respectively. One of the main conclusions was that using the transmittance mode in the NIR region of 740-1000nm was effective for detection of internal defects in both cucumbers and pickles.

In some cases, different works proposed different wavelengths to solve the same problems, such as the detection of defects in certain fruits. This fact seems to indicate that more attention is paid to the statistical techniques or to the capabilities of the technology than to what is really happening in the fruits or vegetables. Results may be influenced by the illumination, the calibration of the system, the variables taken in the statistical models and, in general, by the particular experimental conditions. In this respect, it would be desirable to complete the works with chemical analyses that explain the meaning of the wavelengths found.

\section{Estimation of fruit quality}

Hyperspectral imaging has recently emerged as a powerful inspection tool for quality assessment of fruits and vegetables. The quality of a piece of fruit or vegetable is defined by several attributes that determine its marketability and shelf life. Quality assessment is therefore one of the most important goals of the highly competitive food industry. Product quality includes external appearance, such as colour and the presence of skin diseases or bruises, and internal quality features, including sugar content or firmness. External quality measurements of fruits are currently being determined in many cases by computer vision systems based on colour images. Even though such techniques offer important advantages like real-time operation, lower cost or simulation of human processes, they also have some limitations, the main one being the fact that the human eye is restricted to the visible part of the electromagnetic spectrum and misses important information that is outside these limits. Therefore, to expand quality inspection beyond human limitations, it is necessary to employ instrumental measurements such as hyperspectral imaging (Sun, 2010). One of the applications of these systems is thus the enhancement of the traditional computer vision techniques in the inspection of the external quality by developing more accurate systems for the estimation of particular quality features. However, this technology is being used largely because of the capability of hyperspectral imaging to detect the presence of numerous chemical compositions that can be related with internal quality, as well as estimating their spatial distribution, which cannot be achieved by traditional systems.

\subsection{Estimation of external quality parameters}

Detection of skin defects is one of the most widespread uses of hyperspectral imaging in the inspection of fruits and vegetables, since the perceived quality is highly associated with a good appearance of the product. Many applications aimed at such detection have been reported, mainly in apples. An example of such applications is the detection of bruises on apples. Bruising is one of the major surface defects of apples and they lower the quality of the fruit and entail significant economic losses. Therefore, a great deal of effort has been made to improve bruise detection. Lu (2003) developed a NIR hyperspectral imaging system for detecting both new and old bruises on apples in the 900-1700 nm spectral region. PCA and minimum noise fraction transform (MNF) were performed on the images acquired from Red Delicious and Golden Delicious apples over a period of 47 days after bruising. Results showed that the spectral region between 1000 and $1340 \mathrm{~nm}$ was the most appropriate for bruise detection. Bruise features changed over time from lower reflectance to higher reflectance, and the rate of the change varied with fruit and variety. The system was able to detect both new and old bruises, with a correct detection rate of between $62 \%$ and $88 \%$ for Red Delicious and from $59 \%$ to $94 \%$ for Golden Delicious. This research therefore showed that NIR hyperspectral imaging was useful for detecting apple bruises. Xing and Baerdemaeker (2005) used a hyperspectral imaging system (400-1000 nm) to detect bruises on Jonagold 
apples. Six optimal wavelengths were selected (571, 608, 671, 709, 798 and $867 \mathrm{~nm}$ ) and PCA was then performed on the multispectral image. It was shown that the first PC score images could be used as a good representation of the height profile of the apple surface to determine whether there was shape deformation of the surface, which was assumed to be one feature of the bruised region. The contour plots for the first PC score images were used to distinguish between sound apples and bruised apples, the result being a classification rate for sound apples of $84.6 \%$ and $77.5 \%$ for bruised apples.

Apart from external defects, the presence of contaminants on apples was studied by Mehl et al. (2004), who presented a hyperspectral imaging system as a tool to detect defects and contamination on the surfaces of Red Delicious, Golden Delicious, Gala and Fuji apples, including side rots, bruises, flyspecks, scabs and moulds, fungal diseases (such as black pox) and soil contaminations. Differences in spectral responses within the 430-900 $\mathrm{nm}$ spectral range were analysed using PCA and second difference methods for sorting sound and contaminated apples. An asymmetric second difference method using a chlorophyll absorption waveband at $685 \mathrm{~nm}$ and two bands in the NIR region was tested. This study showed that this asymmetric second difference method provided good detection of the defective/contaminated apples, regardless of the cultivar and the colour of the apple. A hyperspectral NIR imaging system (900-1700 nm) was developed by Nicolaï et al. (2006) to identify bitter pit lesions on apples. A discriminant PLS calibration model was constructed to discriminate between pixels of unaffected apple skin and bitter pit lesions. The system was able to identify bitter pit lesions, even when not visible to the naked eye, such as just after harvest, but could not discriminate between bitter pit lesions and corky tissue. The reduced luminosity at the boundary of the image caused in one image some misclassification errors, which highlights the importance of the lighting system. Ideally, this should be spectrally and spatially uniform, taking into account the geometry of the sample. A flat object is not the same as a spherical one and in many cases corrections are needed (Gómez-Sanchis et al., 2008c).

Other works have also demonstrated the value of hyperspectral imaging for the detection of skin defects and damage in other species of fruits like citrus fruits. Martínez-Usó et al. (2005) used a hyperspectral system (400-720 nm) to develop an unsupervised algorithm capable of segmenting images of oranges for detection of their surface defects. The algorithm employed a minimisation function which took into account the intensity of each band together with edge information. Due to the unsupervised nature of the procedure, it could adapt itself to the large variability of intensities and shapes of the image regions. Thus, this segmentation strategy was able to locate different regions and find their contours satisfactorily. Another hyperspectral imaging approach to defect detection in citrus fruits was described by Qin et al. (2009), who detected canker lesions and other common peel diseases, including greasy spot, insect damage, melanose, scab and wind scar, by means of the spectral information divergence (SID) classification method. This algorithm was based on quantifying the spectral similarities by using a predetermined canker reference spectrum. SID was performed on the hyperspectral images of Ruby Red grapefruits in the spectral region from 450 to $930 \mathrm{~nm}$. The overall classification accuracy was $96.2 \%$, thus demonstrating that hyperspectral imaging coupled with the SID classification method could be used to discriminate citrus canker from other confounding diseases. Blasco et al. (2009) presented a computer vision system that was developed for the recognition and classification of the most common external defects in citrus fruits. Images of oranges and mandarins were acquired using three different systems: visible colour, NIR reflectance and UV-induced fluorescence. The authors used an unsupervised regiongrowing algorithm to segment the image, then an analysis of, morphological parameters of the final regions, and a decision algorithm in order to classify the fruits in categories. The overall success rate reached $86 \%$. However, the greatest increment was achieved in the identification of anthracnose and green mould (95\% and $97 \%$, respectively), which are dangerous defects that spread the disease to sound fruits and their rate of identification must be as high as possible.

A number of hyperspectral imaging methodologies have been researched for the external quality assessment of cucumbers. Cheng et al. (2004) proposed a novel method for hyperspectral feature extraction and applied it to the detection of chilling injury on cucumbers. They used a hyperspectral imaging system with a spectral range of 447-951 nm for tests on cucumbers. This new method combined PCA and Fisher's linear discriminant (FLD) method to show that the hybrid PCA-FLD method maximised the representation and classification effects on the extracted new feature bands. Results showed that this new integrated PCA-FLD method outperformed the PCA and FLD methods when they were used separately for classification, achieving a minimum defect recognition rate of $91 \%$ and a minimum sound cucumber recognition rate of $88.3 \%$ in the worst case. Later, Liu et al. (2005) also used the same hyperspectral imaging system for detecting chilling injury on cucumbers. Images of cucumbers were acquired before and during cold storage treatment as well as during post-chilling RT storage. Both 
simple band ratio algorithms and PCA were tested to discriminate good cucumber skins from those of chilling-injured cucumbers. Results revealed that both the dual-band ratio algorithm, using the 811 and $756 \mathrm{~nm}$ spectral reflectance, and the PCA model from a narrow spectral region of 733-848 nm were able to detect chilling-injured skins of cucumbers within 3-7 days of storage at RT with a success rate of over $90 \%$. On the other hand, chilling injury was relatively difficult to detect at the initial post-chilling RT stage, especially during the first 0-2 days in storage, due to the insignificant manifestation of chillinginduced symptoms. A similar approach was employed in the 900-1700 nm region for detection of bruises on pickling cucumbers by Ariana et al. (2006). PCA, band ratio and band difference were applied in the image processing to segregate bruised cucumbers from normal cucumbers. Best detection accuracies from the PCA were achieved when a bandwidth of $8.8 \mathrm{~nm}$ and the spectral region of 950-1350 nm were selected. It was found that detection accuracy was dependent on the time since bruising. Detection accuracies from the PCA decreased from $95 \%$ to $75 \%$ over the 6-day period after bruising. The best band ratio of 988 and $1085 \mathrm{~nm}$ had detection accuracies of between $93 \%$ and $82 \%$, whereas the best band difference of 1346 and $1425 \mathrm{~nm}$ had accuracies between $89 \%$ and $84 \%$. The general classification performance analysis suggested that band ratio and difference methods offered a similar performance, but they were better than PCA in the estimation of the quality of the pickling cucumbers.

Hyperspectral imaging has been applied to determine the external quality of many other fruits and vegetables. Gowen et al. (2008) investigated the potential application of a hyperspectral imaging system (400-1000 nm) for detecting bruise damage on white mushrooms. Two data reduction methods based on PCA were investigated. After applying PCA, the images obtained from both methods were segmented using the simple thresholding method. Accuracies between $79 \%$ and $100 \%$ were obtained for both methods. Hyperspectral imaging was applied to inspect pears by Zhao et al. (2010), who used a hyperspectral imaging system $(408-1117 \mathrm{~nm})$ for bruise detection on pears. PCA was first applied to process the hyperspectral images in order to obtain the second PC score image, which represented the bruised region best. Then, several classification algorithms were used in a comparative manner to classify pixels into bruised or sound classes, including maximum likelihood, Euclidean distance, Mahalanobis distance (MD), and spectral angle mapper (SAM). Results showed that MD and SAM offered the best performance, with detection accuracies of $93.8 \%$ and $95.0 \%$, respectively. Moreover, compared with the other classification algorithms, MDC and SAM were able to overcome the effects of uneven illumination in detecting bruising of pears by the hyperspectral imaging sensor technique.

Hyperspectral reflectance imaging was used by Karimi et al. (2009) to study the changes in reflectance (350-2500 nm) of avocados coated with different formulations. Wang et al. (2011) used a reflectance hyperspectral system $(400-720 \mathrm{~nm})$ for the detection of external insect damage in jujube fruits. The peel conditions of jujube samples were tested at different undamaged stem-end/calyx-end/cheek regions and at insect-damaged stem-end/cheek regions. A stepwise discriminant analysis was used to classify the jujubes as insect-infested or free of infestation based on the wavelengths that were identified as effective. According to the results, none of the sound cheek or undamaged calyx-end regions were misclassified as having stem-end or insect infestation. Over $98 \%$ of the intact jujubes and $94 \%$ of the insect-infested jujubes represented in the images were recognised correctly, and the overall classification accuracy was about $97 \%$. The results demonstrated that hyperspectral imaging based on a statistically derived discriminant function can be used to discriminate insect infestation from other confounding surface features in jujubes. Moreover, absorbance images (1000-1600 nm) were used by Sugiyama et al. (2010) to discriminate between the skin, stem and leaves of frozen blueberries. The optimal illumination wavelengths for distinguishing foreign materials were determined to be 1268 and $1317 \mathrm{~nm}$, according to the results of a discriminant analysis of absorbance spectra. LDA was used to select the wavelengths and to separate between classes. A threshold was then used to classify the pixels.

Table 2 summarises some of the most important works related with the estimation of external quality using hyperspectral systems.

Table 2. Works related with the estimation of external quality features of fruits

\begin{tabular}{cllc}
\hline \multicolumn{1}{c}{ Reference } & \multicolumn{1}{c}{ Fruit } & \multicolumn{1}{c}{ Features } & Wavelengths \\
\hline Ariana et al. (2006) & Pickling cucumbers & Bruises & 900-1700 nm \\
Blasco et al. (2009) & Oranges and mandarins & $\begin{array}{l}\text { Thrips, phytotoxicity, scarring, scales, } \\
\text { chilling injury, sooty mould, }\end{array}$ & visible, NIR \\
& & $\begin{array}{l}\text { oleocellosis, anthracnose, stem-end } \\
\text { injury, medfly egg deposition and } \\
\text { green mould }\end{array}$ & \\
& & Chilling injury & $447-951 \mathrm{~nm}$
\end{tabular}




\begin{tabular}{|c|c|c|c|}
\hline ElMasry et al. (2008) & McIntosh apples & Bruises & $400-1000 \mathrm{~nm}$ \\
\hline $\begin{array}{l}\text { Gómez-Sanchis et al. } \\
(2008 \mathrm{~b})\end{array}$ & Citrus fruits & Decay & $460-1020 \mathrm{~nm}$ \\
\hline Gowen et al. (2008) & White mushrooms & Bruises & $400-1000 \mathrm{~nm}$ \\
\hline Gowen et al. 2009 & White mushrooms & Freeze damage & $400-1000 \mathrm{~nm}$ \\
\hline Lefcout et al. (2006) & $\begin{array}{l}\text { Golden and Red Delicious } \\
\text { apples }\end{array}$ & Faeces & $465-900 \mathrm{~nm}$ \\
\hline Liu et al. (2005) & Cucumbers & Chilling injury & $447-951 \mathrm{~nm}$ \\
\hline $\mathrm{Lu}(2003)$ & $\begin{array}{l}\text { Red Delicious and Golden } \\
\text { Delicious apples }\end{array}$ & Bruises & $900-1700 \mathrm{~nm}$ \\
\hline Karimi et al. (2009) & Avocados & Reflectance & $350-2500 \mathrm{~nm}$ \\
\hline Martínez-Usó et al. (2005) & Oranges & Skin defects & $400-720 \mathrm{~nm}$ \\
\hline Mehl et al. (2004) & $\begin{array}{l}\text { Red Delicious, Golden } \\
\text { Delicious, Gala, and Fuji } \\
\text { apples }\end{array}$ & $\begin{array}{l}\text { Side rots, bruises, flyspecks, scabs, } \\
\text { moulds, fungal diseases and soil } \\
\text { contaminations }\end{array}$ & $430-900 \mathrm{~nm}$ \\
\hline Qin et al. (2009) & Ruby Red grapefruits & $\begin{array}{l}\text { Canker lesions, greasy spot, insect } \\
\text { damage, melanose, scab and wind scar }\end{array}$ & $450-930 \mathrm{~nm}$ \\
\hline $\begin{array}{l}\text { Xing and Baerdemaeker } \\
(2005)\end{array}$ & Jonagold apples & Bruises & $400-1000 \mathrm{~nm}$ \\
\hline Wang et al. (2011) & Jujubes & Insect damage & $400-720 \mathrm{~nm}$ \\
\hline Sugiyama et al. (2010) & Blueberries & Foreign material & $1000-1600 \mathrm{~nm}$ \\
\hline Zhao et al. (2010) & Pears & Bruises & $408-1117 \mathrm{~nm}$ \\
\hline
\end{tabular}

\subsection{Estimation of internal quality parameters}

Hyperspectral imaging has also been widely used (mostly on apples) to measure internal quality attributes of fruits, such as sugar or SSC, flesh and skin colour, firmness, acidity and starch index, and so forth. Concerning internal quality, maturity is extremely important in the determination of harvest time and the marketing context. Several parameters are normally used to assess fruit maturity, for instance firmness. In recent years, many works on the determination of the maturity of apples have been reported. An example of such studies is that of Peng and $\mathrm{Lu}$ (2005), who developed a method for predicting apple fruit firmness using a multispectral imaging system. A Lorentzian distribution (LD) function with three parameters was proposed to characterise spatial scattering profiles from scattering images for Red Delicious apples at four wavelengths $(680,880,905$ and $940 \mathrm{~nm})$. A multi-linear regression analysis was performed to describe the relationship between parameters of the scattering profile and the firmness of apples. Apple fruit firmness was predicted with a correlation coefficient (r) of 0.82 . This confirmed the feasibility of using an LD function to predict apple firmness. A similar approach was used by $\mathrm{Lu}$ and Peng (2006) for assessing firmness in Red Haven and Coral Star peach fruit, the result being that a wavelength of $677 \mathrm{~nm}$ had the highest correlation with firmness for a single wavelength, although at least 11 wavelengths were needed to obtain better results. Later, an LCTF-based hyperspectral imaging system was used by the same research group (Peng and Lu, 2006) to measure fruit firmness of apples. Spectral images from Red Delicious and Golden Delicious apples were acquired from 650 to $1000 \mathrm{~nm}$. Similarly to their previous work, they used a modified LD function with four parameters, instead of three, to characterise the scattering profiles. In addition, for each wavelength, a multi-linear regression analysis was attempted between firmness and LD parameters for both cultivars. The correlation between LD parameters and fruit firmness was found to change with wavelength; the best single wavelengths were $810 \mathrm{~nm}$ for Red Delicious $(r=0.58)$ and $690 \mathrm{~nm}$ for Golden Delicious $(r=0.50)$. These results indicated that while LD parameters at single wavelengths were related to fruit firmness, they were insufficient for accurate prediction of fruit firmness.

Noh et al. (2007) developed an integrated hyperspectral reflectance and fluorescence imaging technique for measuring apple maturity. Both fluorescence and reflectance scattering images were acquired using a hyperspectral imaging system in the 500-1000 nm region from Golden Delicious apples. Standard destructive tests were performed to measure multiple maturity parameters like flesh and skin colour, firmness, SSC, starch and titratable acid (TA). An approach similar to the one employed in the works mentioned above, using an LD function and multi-linear regression analysis, was employed to relate LD parameters to individual maturity parameters for each sensing mode and their combined data. Overall, the fluorescence prediction models had consistently lower correlations with individual maturity parameters than the reflectance models. Excellent predictions of skin and flesh hue were obtained $(r \geq 0.90)$ with either the fluorescence models or the reflectance models. However, the fluorescence and reflectance models both yielded poorer prediction results for TA. The integration of reflectance and fluorescence improved maturity measurements over either reflectance or fluorescence alone; the improvements in correlation were noticeable for most parameters (up to $10.6 \%$ for TA). Similarly, Noh and Lu (2007) also employed laser-induced fluorescence scattering images to predict multiple quality parameters (fruit skin 
and flesh colour, firmness, SSC and TA). Fluorescence scattering images were acquired from Golden Delicious apples by a hyperspectral imaging system $(500-1000 \mathrm{~nm})$. The apples were illuminated by a continuous-wave blue (408 nm) laser at six different excitation times. A hybrid method of combining PCA and ANN modelling was used to predict fruit quality parameters. The differences were minimal in the model prediction results from the fluorescence data obtained with illumination at 1, 2, 3, 4 and $5 \mathrm{~m}$; therefore, fluorescence could be performed within $1 \mathrm{~m}$ of illumination. Excellent predictions were obtained for apple skin hue with $\mathrm{r}=0.94$ and relatively good predictions were obtained for fruit firmness, skin chroma and flesh hue $(r \geq 0.74)$. On the other hand, poorer correlations were found for SSC, TA and flesh chroma. Zhao et al. (2009) applied hyperspectral imaging (408-1117 nm) to determine the sugar content of apple cv. Fuji. On applying the PLS method to the spectral profiles of the fruits, it was found that the optimal spectral range for sugar content was 704-805 $\mathrm{nm}$. The authors found that PLS analysis gave a reasonably good correlation for estimating the sugar content of apple $(r=0.91)$.

Despite the importance of the parameters mentioned above in the measurement of fruit maturity, the most reliable maturity index is the starch degradation pattern of the pulp. Apple fruit maturation is characterised by an almost complete conversion of starch into sugars. The most usual way to assess the starch conversion stage is the iodine test, in which cut fruit is dipped in iodine solution and stain patterns are visually evaluated by inspectors comparing them with reference charts. However, of late researchers have been focusing on developing non-destructive techniques to assess the starch index that avoid subjective assessment and the usage of toxic iodine solution. Peirs et al. (2003) used a hyperspectral imaging system $(868-1789 \mathrm{~nm})$ to measure the starch distribution and starch index of apple fruit during maturation. Using PCA on the hyperspectral images, four wavelengths were selected $(989,1051,1131$ and $1215 \mathrm{~nm}$ ) and PCA was then applied again to the multispectral images. A threshold value was defined to classify the pixels of the first PC score image of an apple into pixels containing a concentration higher or lower than the threshold value for staining. Hence, these images confirmed the spatial starch degradation pattern as determined by the traditional iodine technique.

Another hyperspectral imaging approach to assess the starch index is the one described by Menesatti et al. (2009). They used visible and NIR images of Golden Delicious apples in the spectral region between 1000 and $1700 \mathrm{~nm}$. They studied the relationships between NIR spectral images, colour images and visually assessed starch/starch-free patterns, indirectly measuring the stages of apple maturity. PLSDA was used on hyperspectral NIR images to classify single pixels in two classes (starch and starch-free), achieving 66\% accuracy. Other applications of hyperspectral imaging systems to the assessment of the internal quality of apples have been cited in recent literature, such as chilling injury detection. Damage in fruit cell membranes due to chilling injury affects normal firmness, and therefore changes in firmness could be used as an indication of possible chilling injury. The starch index was also employed by Nguyen Do Trong et al. (2011) to estimate the optimal cooking time of potatoes. The changes in the microstructure during cooking affect the interaction of light with the starch granules in different regions inside the potato. The potential of hyperspectral imaging (400-1000 nm) was studied for contactless detection of the cooking front in potatoes. PLSDA was used to discriminate between the pixel spectra for the cooked regions and those for the regions that remained raw. By modelling the evolution of the cooking front over time, the optimal cooking time could be predicted with less than $10 \%$ relative error. ElMasry et al. (2009) detected chilling injury and predicted firmness in Red Delicious apples using a hyperspectral imaging system (400-1000 nm) and ANN techniques. Experimental results demonstrated that a spectral imaging system associated with ANN could successfully distinguish between chillinginjured apples and normal apples (98.4\% accuracy), as well as detect changes in firmness $(\mathrm{r}=0.92)$.

Hyperspectral imaging has also been used for determining the internal quality of other fruits and vegetables, apart from apples. Qin and $\mathrm{Lu}$ (2005) used hyperspectral transmission images in the 400$1000 \mathrm{~nm}$ spectral region to detect pits in tart cherries using ANN to classify cherries with and without pits. Experiments resulted in low average classification errors (about 3\%). It was shown that sample orientation and colour did not significantly affect classification accuracy, but the size of the fruit did. ElMasry et al. (2007) determined MC, SSC and acidity in strawberries by means of a visible-NIR hyperspectral imaging system $(400-1000 \mathrm{~nm})$, with results that showed a good prediction performance for moisture content $(\mathrm{r}=0.91)$, SSC $(\mathrm{r}=0.80)$ and $\mathrm{pH}(\mathrm{r}=0.94)$. Moreover, for classifying strawberries according to the stage of their ripeness, a texture analysis was conducted on the images based on greylevel co-occurrence matrix (GLCM) parameters, a maximum classification accuracy of $90 \%$ being achieved. Fernandes et al. (2011) reported a system based on neural networks for the estimation of grape anthocyanin concentration using hyperspectral images (400-1000 nm). They used a method based on NN called AdaBoost. The inputs from the NN were the PCs of the spectra of the grapes. Hyperspectral data 
were collected in the reflectance mode for 46 individual whole grapes of the Cabernet Sauvignon variety. The anthocyanin concentration values obtained by expert calibrations had a squared correlation coefficient value of 0.65 compared to the values measured using conventional laboratory techniques. The internal quality of whole pickles was studied by Ariana and Lu (2010b) using transmittance (675-1000 $\mathrm{nm})$ and reflectance (400-675 $\mathrm{nm}$ ) hyperspectral imaging systems. Images of pickles were obtained using a prototype in-line hyperspectral imaging system. PCA was applied to the hyperspectral images of the pickle samples for the detection of bloater damage. Transmittance images were far more effective for internal defect detection than reflectance ones. An overall defect classification accuracy of $86 \%$ was achieved, compared with an accuracy of $70 \%$ by the human inspectors.

Banana fruit quality and maturity stages were studied by Rajkumar et al. (2011) at three different temperatures $\left(20,25\right.$ and $\left.30^{\circ} \mathrm{C}\right)$ by using a hyperspectral imaging technique in the visible and NIR (400$1000 \mathrm{~nm}$ ) regions. The quality parameters like MC, firmness and SSC were determined and correlated with the spectral data using PLS. The model showed that 10 latent factors could be used to select the optimal wavelength based on beta coefficients. Eight wavelengths were required to predict the maturity stages of banana fruits representing the quality attribute in terms of the features that were studied.

Table 3 summarises some of the most important works related with the estimation of the internal quality of fruits using hyperspectral systems.

Table 3. Works related with the estimation of internal quality features of fruits

\begin{tabular}{|c|c|c|c|}
\hline Reference & Fruit & $\begin{array}{r}\text { Features } \\
\end{array}$ & Wavelengths \\
\hline ElMasry et al. (2007) & Strawberries & MC, SSC and acidity & $400-1000 \mathrm{~nm}$ \\
\hline ElMasry et al. (2009) & Red Delicious apples & Chilling injury, firmness & $400-1000 \mathrm{~nm}$ \\
\hline Menesatti et al. (2009) & Golden Delicious apples & Starch & $1000-1700 \mathrm{~nm}$ \\
\hline Noh and Lu (2007) & Golden Delicious apples & $\begin{array}{l}\text { Flesh and skin colour, firmness, } \\
\text { SSC and TA }\end{array}$ & $500-1000 \mathrm{~nm}$ \\
\hline Rajkumar et al. (2011) & Banana & MC, SSC and firmness & $400-1000 \mathrm{~nm}$ \\
\hline Noh et al. (2007) & Golden Delicious apples & $\begin{array}{l}\text { Flesh and skin colour, firmness, } \\
\text { SSC, starch and TA }\end{array}$ & $500-1000 \mathrm{~nm}$ \\
\hline Peirs et al. (2003) & Apple & Starch distribution and index & $868-1789 \mathrm{~nm}$ \\
\hline Peng and $\mathrm{Lu}(2005)$ & Red Delicious apple & Firmness & $\begin{array}{r}680,880,905 \\
\text { and } 940 \mathrm{~nm}\end{array}$ \\
\hline Peng and $\mathrm{Lu}(2006)$ & $\begin{array}{l}\text { Red Delicious and Golden } \\
\text { Delicious apples }\end{array}$ & Firmness & $650-1000 \mathrm{~nm}$ \\
\hline Fernandes et al. (2011) & Grapes & Anthocyanin concentration & $400-1000 \mathrm{~nm}$ \\
\hline Ariana and Lu (2010b) & Pickles & Bloated & $675-1000 \mathrm{~nm}$ \\
\hline Qin and Lu (2005) & Tart cherries & Pits & $400-1000 \mathrm{~nm}$ \\
\hline Zhao et al. (2009) & Fuji apples & Sugar content & $408-1117 \mathrm{~nm}$ \\
\hline
\end{tabular}

\section{Future trends}

The future of hyperspectral systems applied to food inspection is promising, since both the industry and consumers are becoming increasing aware of need to ensure the quality and safety of food, and this technology is an important tool for the automatic inspection and monitoring of these parameters. The price of the equipment is constantly decreasing, while the technology allows more accurate imaging systems to be developed that are capable of going further into the electromagnetic spectrum. This would enable researchers to create new applications oriented towards the non-destructive estimation of internal compounds related with the organoleptic quality or shelf life of the products.

However, there are still two challenges to be overcome using this technology. On the one hand, the acquisition and processing times of the images are still slow, which prevents widespread implementation in an industry that needs real-time inspection. The partial solution is to search for a small set of important wavelengths that can be used to deal with each problem individually but which sometimes miss important information or limit the potential scope of the final application. On the other hand, most of the research being conducted is aimed at detecting these sets of wavelengths or obtaining results to relate with particular objectives. But in most cases results are dependent on the laboratory conditions (lighting, calibration, etc.) or on the statistical techniques used, and are not truly related with internal compounds or physical-chemical properties that could support these results from the product point of view. In these cases, for example, different studies can obtain different sets of wavelengths for similar applications. 


\section{Conclusions}

This paper has summarised the current state of the art on the application of hyperspectral imaging for fruit and vegetable inspection. Most of works deal with statistical techniques to reduce the dimensionality of the problem, being the most used based on ANN, PCA, PLS or LDA. Using the whole captured spectrum or reducing the information to a few bands, the ultimate aim is the inspection of quality beyond the possibilities of traditional computer vision systems based on colour images. However, there are still challenges in this topic that have to be overcome by researchers. Although nowadays these systems fits probably better with laboratory developments, many current works try to provide the industry with important practical solutions. However, very few of them investigate the physical-chemical and biological phenomena that are evidenced in the images. Thus, different works provide very different results for similar problems, for instance in the selection of particular wavelengths. The increasing interdisciplinary nature of research groups offers the possibility of combining genetic, biological and physiological knowledge with physics and computer vision research to take an important step towards integrated solutions for the fruit and vegetable industry. These solutions will not only allow problems to be detected, but will also afford the generation of tools with which to prevent their causes. In general, this is a technology whose use is beginning to extend to inspect the external and internal quality of many horticultural products, mainly because of the constant price reduction of the components and the increment in computation capacity of modern computers. However, its potential as non-destructive analytical tool is not fully exploited and much remains to be investigated.

\section{Acknowledgement}

This work was partially funded by the Instituto Nacional de Investigación y Tecnologia Agraria y Alimentaria de España (INIA) through research project RTA2009-00118-C02-01 and by the Ministerio de Ciencia e Innovación de España (MICINN) through research project DPI2010-19457, both projects with the support of European FEDER funds.

\section{References}

Aleixos N, Blasco J, Navarrón F \& Moltó E (2002). Multispectral inspection of citrus in real-time using machine vision and digital signal processors. Computers and Electronics in Agriculture, 33(2), 121-137.

Al-Mallahi A, Kataoka T \& Okamoto H (2008) Discrimination between potato tubers and clods by detecting the significant wavebands. Biosystems Engineering, 100(3), 329-337.

Ariana DP, Guyer DE \& Shrestha B (2006a) Integrating multispectral reflectance and fluorescence imaging for defect detection on apples. Computers and Electronics in Agriculture, 50, 148-161.

Ariana DP, Lu R \& Guyer DE (2006b) Near-infrared hyperspectral reflectance imaging for detection of bruises on pickling cucumbers. Computers and Electronics in Agriculture, 53, 60-70.

Ariana DP \& Lu R (2010a) Hyperspectral waveband selection for internal defect detection of pickling cucumbers and whole pickles. Computers and Electronics in Agriculture, 74(1), 137-144.

Ariana DP \& Lu R (2010b) Evaluation of internal defect and surface color of whole pickles using hyperspectral imaging. Journal of Food Engineering, 96(4), 583-590.

Bearman G \& Levenson R (2003) Biological imaging spectroscopy. In Biomedical Photonics Handbook Vo-Dinh T (ed), CRC Press, Boca Raton, FL, USA.

Bei L, Dennis GI, Miller HM, Spaine TW \& Carnahan JW (2004) Acousto-optic tunable filters: fundamentals and applications as applied to chemical analysis techniques. Progress in Quantum Electronics, 28(2), 67-87.

Bennedsen BS \& Peterson DL (2005) Performance of a system for apple surface defect identification in near-infrared images. Biosystems Engineering, 90(4), 419-431.

Bennedsen BS, Peterson DL \& Tabb A (2005). Identifying defects in images of rotating apples. Computers and Electronics in Agriculture, 48, 92-102.

Blasco J, Aleixos N, Gómez J \& Moltó E (2007) Citrus sorting by identification of the most common defects using multispectral computer vision. Journal of Food Engineering, 83(3), 384-393.

Blasco J, Aleixos N, Gómez-Sanchis J \& Moltó E (2009). Recognition and classification of external skin damage in citrus fruits using multispectral data and morphological features. Biosystems Engineering, 103, 137-145. 
Cayuela JA, García-Martos JM \& Caliani N (2009) NIR prediction of fruit moisture, free acidity and oil content in intact olives. Grasas y Aceites, 60(2), 194-202.

Chang C (1976) Acousto-optic devices and applications. IEEE Transactions on Sonics Ultrasound, 23(1), $2-22$.

Cheng X, Chen Y, Tao Y, Wang C, Kim MS \& Lefcourt A (2004) A novel integrated PCA and FLD method on hyperspectral image feature extraction for cucumber chilling damage inspection. Transactions of ASAE, 47(4), 1313-1320.

Cubero S, Aleixos N, Moltó E, Gómez-Sanchis J \& Blasco J (2011) Advances in machine vision applications for automatic inspection and quality evaluation of fruits and vegetables. Food and Bioprocess Technology, 4(4), 487-504.

Du C-J \& Sun D-W (2009) Retrospective shading correlation of confocal laser scanning microscopy beef images for three-dimensional visualization. Food and Bioprocess Technology, 2, 167-176.

Egmont-Petersen M, de Ridder D \& Handels H (2002). Image processing with neural networks - a review. Pattern Recognition, 35(10), 2279-2301.

ElMasry G, Wang N, ElSayed A \& Ngadi M (2007) Hyperspectral imaging for nondestructive determination of some quality attributes for strawberry. Journal of Food Engineering, 81, 98-107.

ElMasry G, Wang N, Vigneault C, Qiao J \& ElSayed A (2008) Early detection of apple bruises on different background colors using hyperspectral imaging. LWT 41, 337-345.

ElMasry G, Wang N \& Vigneault C (2009) Detecting chilling injury in Red Delicious apple using hyperspectral imaging and neural networks. Postharvest Biology and Technology, 52, 1-8.

Farrera-Rebollo RR, Salgado-Cruz MP, Chanona-Pérez J, Gutiérrez-López GF, Alamilla-Beltrán L \& Calderón-Domínguez G (2011). Evaluation of Image Analysis Tools for Characterization of Sweet Bread Crumb Structure. Food and Bioprocess Technology. DOI: 10.1007/s11947-011-0513-y, in-press

Fernandes AM, Oliveira P, Moura JP, Oliveira AA, Falco V, Correia MJ \& Melo-Pinto P (2011) Determination of anthocyanin concentration in whole grape skins using hyperspectral imaging and adaptive boosting neural networks. Journal of Food Engineering, 105(2), 216-226.

Fisher R (1936) The use of multiple measurements in taxonomic problems. Annals of Eugenics, 7, 179188.

Gómez-Sanchis J, Camps-Valls G, Moltó E, Gómez-Chova L, Aleixos N \& Blasco J (2008a) Segmentation of hyperspectral images for the detection of rotten mandarins, Lecture Notes in Computer Science, 5112, 1071-1080

Gómez-Sanchis J, Gómez-Chova L, Aleixos N, Camps-Valls G, Montesinos-Herrero C, Moltó E \& Blasco J (2008b) Hyperspectral system for early detection of rottenness caused by Penicillium digitatum in mandarins. Journal of Food Engineering, 89(1), 80-86.

Gómez-Sanchis J, Moltó E, Camps-Valls G, Gómez-Chova L, Aleixos N \& Blasco J (2008c) Automatic correction of the effects of the light source on spherical objects. An application to the analysis of hyperspectral images of citrus fruits. Journal of Food Engineering, 85(2), 191-200.

Gowen AA, O'Donnell CP, Cullen PJ, Downey G \& Frias JM (2007) Hyperspectral imaging - an emerging process analytical tool. Trends in Food Science \& Technology, 18(12), 590-598.

Gowen AA, O'Donnell CP, Taghizadeh M, Cullen PJ, Frias JM \& Downey G (2008) Hyperspectral imaging combined with principal component analysis for bruise damage detection on white mushrooms (Agaricus bisporus). Journal of Chemometrics, 22(3-4), 259-267.

Gowen AA, Taghizadeh M \& O’Donnell CP (2009a) Identification of mushrooms subjected to freeze damage using hyperspectral imaging. Journal of Food Engineering, 93, 7-12.

Gowen AA, Tsenkova R, Esquerre C, Downey G \& O'Donnell PD (2009b) Use of near infrared hyperspectral imaging to identify water matrix co- ordinates in mushrooms (Agaricus bisporus) subjected to mechanical vibration. Journal of Near Infrared Spectroscopy, 17(6), 363-371.

Guyon I \& Elisseeff A (2003) An introduction to variable and feature selection. Journal of Machine Learning Research, 3, 1157-1182. 
Huang Y, Kangas LJ \& Rasco BA (2007) Applications of artificial neural networks (ANNs) in food science. Critical Reviews in Food Science and Nutrition, 47(2), 113-126.

Jiménez A, Beltrán G, Aguilera MP \& Uceda M (2008) A sensor-software based on artificial neural network for the optimization of olive oil elaboration process. Sensors and Actuators B, 129, 985-990.

Jobson JD (1992) Applied Multivariate data Analysis: Categorical and Multivariate Methods Vol. 2 Springer-Verlag, Berlin, Germany.

Jolliffe IT (2002) Principal component analysis, second edition. Springer-Verlag, New York, USA.

Kalkan H, Beriat P, Yardimci Y \& Pearson TC (2011) Detection of contaminated hazelnuts and ground red chili pepper flakes by multispectral imaging. Computers and Electronics in Agriculture, in-press, doi:10.1016/j.compag.2011.03.005.

Karimi Y, Maftoonazad N, Ramaswamy HS, Prasher SO \& Marcotte M (2009) Application of hyperspectral technique for color classification avocados subjected to different treatments. Food and Bioprocess Technology, DOI: 10.1007/s11947-009-0292-x, in press.

Kim MS, Chen YR \& Mehl PM (2001) Hyperspectral reflectance and fluorescence imaging system for food quality and safety. Transactions of the ASAE, 44(3), 721-729.

Kleynen O, Leemans V \& Destain MF (2005) Development of a multi-spectral vision system for the detection of defects on apples. Journal of Food Engineering, 69, 41-49.

Lee JA \& Verleysen M (2007) Nonlinear dimensionality reduction. Springer-Verlag, New York, USA.

Lefcout AM, Kim MS, Chen Y-R \& Kang B (2006) Systematic approach for using hyperspectral imaging data to develop multispectral imagining systems: Detection of feces on apples. Computers and Electronics in Agriculture, 54, 22-35.

Liu Y, Chen YR, Wang CY, Chan DE \& Kim MS (2006) Development of hyperspectral imaging technique for the detection of chilling injury in cucumbers; spectral and image analysis. Applied engineering in agriculture, 22(1), 101-111.

Lleó L, Barreiro P, Ruiz-Altisent M \& Herrero A (2009) Multispectral images of peach related to firmness and maturity at harvest. Journal of Food Engineering, 93(2), 229-235.

Lleó L, Roger JM, Herrero-Langreo A, Diezma-Iglesias B \& Barreiro P (2011) Comparison of multispectral indexes extracted from hyperspectral images for the assessment of fruit ripening. Journal of Food Engineering, 104(4), 612-620.

Lu R (2003) Detection of bruises on apples using near-infrared hyperspectral imaging. Transactions of the ASAE, 523-530.

Lu R \& Peng Y (2006) Hyperspectral Scattering for assessing Peach Fruit Firmness. Biosystems Engineering, 93(2), 161-171.

Manickavasagan A, Jayas DS, White NDG \& Paliwal J (2010) Wheat Class Identification Using Thermal Imaging. Food and Bioprocess Technology, 3(3), 450-460.

Martinez AM \& Kak AC (2004) PCA versus LDA. IEEE Transactions on Pattern Analysis and Machine Intelligence, 23(2), 228-233.

Mather PM (1998) Computer processing of remotely sensed images. John Wiley \& Sons, Chichester, UK.

McLachlan GJ (2004) Discriminant analysis and statistical pattern recognition Wiley-Interscience, New Jersey, USA.

Mehl PM, Chen YR, Kim MS \& Chan DE (2004) Development of hyperspectral imaging technique for detection of apple surface defects and contaminations. Journal of Food Engineering, 61. 67-81.

Menesatti P, Zanella A, D’Andrea S, Costa C, Paglia G, \& Pallottino F (2009) Supervised multivariate analysis of hyper-spectral NIR images to evaluate the starch index of apples. Food and Bioprocess Technology, 2, 308-314.

Nguyen Do Trong N, Tsuta M, Nicolaï BM, De Baerdemaeker J \& Saeys W (2011) Prediction of optimal cooking time for boiled potatoes by hyperspectral imaging. Journal of Food Engineering, 105(4), 617624. 
Nicolaï BM, Lötze E, Peirs A, Scheerlinck N \& Theron KI (2006) Non-destructive measurement of bitter pit in apple fruit using NIR hyperspectral imaging. Postharvest Biology and Technology, 40, 1-6.

Nicolaï BM, Beullens K, Bobelyn E, Peirs A, Saeys W, Theron KI \& Lammertyn J (2007) Nondestructive measurement of fruit and vegetable quality by means of NIR spectroscopy: A review. Postharvest Biology and Technology, 46(2), 99-118.

Noh H, Peng Y \& Lu R (2007) Integration of hyperspectral reflectance and fluorescence imaging for assessing apple maturity. Transactions of the ASABE, 50(3), 963-971.

Noh HK \& Lu R (2007) Hyperspectral laser-induced fluorescence imaging for assessing apple fruit quality, Postharvest Biology and Technology, 43, 193-201.

Ozaki Y, McClure WF \& Christy AA (ed) (2006) Near-infrared spectroscopy in food science and technology. Wiley-Interscience, New Jersey, USA.

Paulus I, De Busscher R \& Schrevens E (1997) Use of image analysis to investigate human quality classification of apples. Journal Agricultural Engineering Research, 68, 341-353.

Peirs A, Scheerlinck N, De Baerdemaeker J \& Nicolaï BM (2003) Starch index determination of apple fruit by means of a hyperspectral near infrared reflectance imaging system. Journal of near infrared spectroscopy, 11(5), 379-389.

Peng Y \& Lu R (2005) Modeling multispectral scattering profiles for prediction of apple fruit firmness. Transactions of the ASAE, 48(1), 235-242.

Peng Y \& Lu R (2006) An LCTF-based multispectral imaging system for estimation of apple fruit firmness; part i. acquisition and characterization of scattering images. Transactions of the ASAE, 49(1), 259-267.

Plaza A, Benediktsson JA, Boardman JW, Brazile J, Bruzzone L, Camps-Valls G, Chanussot J, Fauvel M, Gamba P, Gualtieri A, Marconcini M, Tilton JC \& Trianni G (2009) Recent advances in techniques for hyperspectral image processing. Remote Sensing of Environment, 113 (1), S110-S122.

Polder G, van der Heijden GWAM \& Young IT (2002) Spectral image analysis for measuring ripeness of tomatoes. Transactions of the ASAE, 45, 1155-1161.

Polder G, van der Heijden GWAM \& Young IT (2003) Tomato sorting using independent component analysis on spectral images. Real-Time Imaging, 9, 253-259.

Polder G, van der Heijden GWAM, van der Voet H \& Young IT (2004) Measuring surface distribution of carotenes and chlorophyll in ripening tomatoes using imaging spectrometry. Postharvest Biology and Technology, 34, 117-129.

Qin J \& Lu R (2005) Detection of pits in tart cherries by hyperspectral transmission imaging. Transactions of the ASAE, 48(5), 1963-1970.

Qin J, Burks TF, Ritenour MA \& Bonn WG (2009) Detection of citrus canker using hyperspectral reflectance imaging with spectral information divergence. Journal of Food Engineering, 93, 183-191.

Quevedo R, Aguilera JM \& Pedreschi F (2010) Color of salmon fillets by computer vision and sensory panel. Food and Bioprocess Technology, 3(5), 637-643.

Quevedo R \& Aguilera (2010) Color computer vision and stereoscopy for estimating firmness in the salmon (Salmon salar) fillets, Food and Bioprocess Technology, 3(4), 561-567.

Rajkumar P, Wang N, EImasry G, Raghavan GSV \& Gariepy Y (2011) Studies on banana fruit quality and maturity stages using hyperspectral imaging. Journal of Food Engineering, DOI: 10.1016/ j.jfoodeng.2011.05.002, in-press.

Shaw PJA (2003) Multivariate statistics for the environmental sciences, Hodder-Arnold, New York, USA.

Shih FY (2010) Image processing and pattern recognition: fundamentals and techniques, Wiley-IEEE Press, New York, USA.

Sjöström M, Wold S \& Söderström B (1986) PLS discriminant plots. In: Gelsema ES \& Kanal LN (ed) Pattern Recognition in Practice II, pp 461-470, Elsevier, Amsterdam, (The Netherlands). 
Sugiyama T, Sugiyama J, Tsuta M, Fujita K, Shibata M, Kokawa M, Araki T, Nabetani H \& Sagara Y (2010) NIR spectral imaging with discriminant analysis for detecting foreign materials among blueberries. Journal of Food Engineering, 101(3), 244-252.

Sun D-W (ed) (2007) Computer vision technology for food quality evaluation. Academic Press, Elsevier Science, London, UK.

Sun D-W (ed) (2009) Infrared spectroscopy for food quality analysis and control. Academic Press, Elsevier Science, London, UK.

Sun D-W (ed) (2010) Hyperspectral imaging for food quality analysis and control. Academic Press, Elsevier Science, London, UK.

Taghizadeh M, Gowen AA, Ward P \& O'Donnell CP (2010) Use of hyperspectral imaging for evaluation of the shelf-life of fresh white button mushrooms (Agaricus bisporus) stored in different packaging films. Innovative Food Science \& Emerging Technologies, 11(3), 423-431.

Taghizadeh M, Gowen AA \& O'Donnell CP (2011) Comparison of hyperspectral imaging with conventional RGB imaging for quality evaluation of Agaricus bisporus mushrooms. Biosystems Engineering, 108 (2), 191-194

Taghizadeh M, Gowen AA \& O'Donnell CP (2011) The potential of visible-near infrared hyperspectral imaging to discriminate between casing soil, enzymatic browning and undamaged tissue on mushroom (Agaricus bisporus) surfaces. Computers and Electronics in Agriculture, 77(1), 74-80.

Unay D \& Gosselin B (2006) Automatic defect segmentation of 'Jonagold' apples on multi-spectral images: A comparative study. Postharvest Biology and Technology, 42, 271-79.

Unay D, Gosselin B, Kleynen O, Leemans V, Destain MF \& Debeir O (2011) Automatic grading of Bicolored apples by multispectral machine vision. Computers and Electronics in Agriculture, 75(1), 204212.

Vila J, Calpe J, Pla F, Gómez L, Connell J, Marchant JA, Calleja J, Mulqueen M, Muñoz J \& Klaren AC (2005) SmartSpectra: Applying multispectral imaging to industrial environments. Real-Time Imaging, 11, 85-98.

Vila-Francés J, Calpe-Maravilla J, Gómez-Chova L \& Amorós-López J (2010) Analysis of acousto-optic tunable filter performance for imaging applications. Optical Engineering, 49(11), 113203-113203-9.

Vila-Francés J, Calpe-Maravilla J, Gómez-Chova L \& Amorós-López J (2011) Design of a configurable multispectral imaging system based on an AOTF. IEEE Transactions on Ultrasonics, Ferroelectrics and Frequency Control, 58(1), 259-262.

Vinzi V, Chin WW, Henseler J \& Wang H (ed) (2010) Handbook of partial least squares. SpringerVerlag, Berlin, Germany.

Wang J, Nakano K, Ohashi S, Kubota Y, Takizawa K \& Sasaki Y (2011) Detection of external insect infestations in jujube fruit using hyperspectral reflectance imaging. Biosystems Engineering, 108(4), 345351.

Xing J \& De Baerdemaeker J (2005) Bruise detection on 'Jonagold' apples using hyperspectral imaging. Postharvest Biology and Technology, 37(2), 152-162.

Xing J, Bravo C, Jancsók PT, Ramon H \& De Baerdemaeker J (2005) Detecting bruises on 'golden delicious' apples using hyperspectral imaging with multiple wavebands. Biosystems Engineering, 90(1), 27-36.

Xing J, Jancsók PT \& De Baerdemaeker J (2007) Stem-end/calyx identification on apples using contour analysis in multispectral images. Biosystems Engineering, 96(2), 231-237.

Xing J, Saeys W \& De Baerdemaeker J (2007) Combination of chemometric tools and image processing for bruise detection on apples. Computers and Electronics in Agriculture, 56(1), 1-13.

Zhao J, Vittayapadung S, QuanSheng C, Chaitep S \& Chuaviroj, R (2009) Nondestructive measurement of sugar content of apple using hyperspectral imaging technique. Maejo International Journal of Science and Technology, 3(1), 130-142. 